\title{
The barycenter method on singular spaces
}

\author{
Peter A. Storm*
}

\begin{abstract}
Compact convex cores with totally geodesic boundary are proven to uniquely minimize volume over all hyperbolic 3-manifolds in the same homotopy class. This solves a conjecture in Kleinian groups concerning acylindrical 3-manifolds. Closed hyperbolic manifolds are proven to uniquely minimize volume over all compact hyperbolic cone-manifolds in the same homotopy class with cone angles $\leq 2 \pi$. Closed hyperbolic manifolds are proven to minimize volume over all compact Alexandrov spaces with curvature bounded below by -1 in the same homotopy class. A version of the Besson-Courtois-Gallot theorem is proven for $n$-manifolds with boundary. The proofs extend the techniques of Besson-Courtois-Gallot.
\end{abstract}

\section{Introduction}

This paper extends the barycenter map machinery of Besson-Courtois-Gallot [BCG1] to a class of singular metric spaces called convex Riemannian amalgams (defined in Section 2.8). This class of singular spaces includes cone-manifolds and the metric doubling of hyperbolic convex cores across their boundary. These singular space techniques are used to solve a conjecture in Kleinian groups due to Bonahon. Specifically, we prove that compact convex cores with totally geodesic boundary uniquely miminize volume over all hyperbolic 3-manifolds in the same homotopy class (see Theorem 8.1).

The main tool of this paper is the following extension of Besson-Courtois-Gallot techniques to convex Riemannian amalgams.

Theorem 4.1. For $n \geq 3$, let $Z$ be a compact $n$-dimensional convex Riemannian amalgam. Let $M_{\mathrm{hyp}}$ be a closed hyperbolic $n$-manifold. Let $h(\widetilde{Z})$ denote the volume growth entropy of the universal cover of $Z$. If $f: Z \rightarrow M_{\text {hyp }}$ is a homotopy equivalence then

$$
h(\widetilde{Z})^{n} \operatorname{Vol}(Z) \geq(n-1)^{n} \operatorname{Vol}\left(M_{\mathrm{hyp}}\right)
$$

with equality if and only if $f$ is homotopic to a homothetic homeomorphism.

By restricting attention to cone-manifolds, we obtain

\footnotetext{
*This research was partially supported an NSF Postdoctoral Fellowship.
} 
Theorem 8.6. For $n \geq 3$, let $Z$ be compact $n$-dimensional cone-manifold built with simplices of constant curvature $K \geq-1$. Assume all its cone angles are $\leq 2 \pi$. Let $M_{\mathrm{hyp}}$ be a closed hyperbolic n-manifold. If $f: Z \rightarrow M_{\mathrm{hyp}}$ is a homotopy equivalence then

$$
\operatorname{Vol}(Z) \geq \operatorname{Vol}\left(M_{\text {hyp }}\right)
$$

with equality if and only if $f$ is homotopic to an isometry.

If $Z$ is allowed to be any Alexandrov space with curvature bounded below by -1 (see Section 2.13), we then obtain

Theorem 8.7. Let $Z$ be a compact $n$-dimensional $(n \geq 3)$ Alexandrov space with curvature bounded below by -1 . Let $M_{\text {hyp }}$ be a closed hyperbolic n-manifold. If $f: Z \rightarrow M_{\text {hyp }}$ is a homotopy equivalence then

$$
\operatorname{Vol}(Z) \geq \operatorname{Vol}\left(M_{\text {hyp }}\right)
$$

As mentioned above, the paper's main theorems solve a conjecture in Kleinian groups. To state things precisely, let $\mathrm{H}(N)$ denote the set of marked oriented isometry classes of hyperbolic 3-manifolds $M$ equipped with a homotopy equivalence $N \rightarrow M$. Define a volume function

$$
\text { Vol: } M \in \mathrm{H}(N) \mapsto \operatorname{Vol}\left(C_{M}\right) .
$$

It is a consequence of Thurston's Geometrization Theorem and Mostow Rigidity that $N$ is acylindrical if and only if there exists a convex cocompact $M_{g} \in \mathrm{H}(N)$ such that $\partial C_{M_{g}}$ is totally geodesic [Th2, p. 14]. Moreover, $M_{g}$ is unique up to isometry. Let $M_{g}^{\mathrm{opp}}$ denote $M_{g}$ with the opposite orientation.

Conjecture. $M_{g}$ and $M_{g}^{\text {opp }}$ are the only global minima of Vol over $\mathrm{H}(N)$.

Initial progress on this conjecture was made by Bonahon [Bon]. Using different methods, Bonahon proved that $M_{g}$ is a strict local minimum of Vol (in the quasiisometric topology on $\mathrm{H}(N))$. In [S], the author proved that $M_{g}$ and $M_{g}^{\mathrm{opp}}$ are global minima of Vol. Here this conjecture is completely solved by

Theorem 8.1. Let $N$ be a compact acylindrical 3-manifold. Let $M_{g} \in \mathrm{H}(N)$ be a convex cocompact hyperbolic 3-manifold such that the boundary of the convex core $\partial C_{M_{g}} \subset M_{g}$ is totally geodesic. Then for all $M \in \mathrm{H}(N)$,

$$
\operatorname{Vol}\left(C_{M}\right) \geq \operatorname{Vol}\left(C_{M_{g}}\right)
$$

with equality if and only if $M$ and $M_{g}$ are isometric.

The techniques used to prove Theorem 8.1 immediately generalize to a version of the Besson-Courtois-Gallot theorem for manifolds with boundary. (Perelman's 
unpublished Doubling theorem [P, Theorem 5.2] is used in the proof of Theorem 8.9. See Theorem 8.8.)

Theorem 8.9. Let $Z$ be a compact convex Riemannian n-manifold with boundary $(n \geq 3)$. Assume the sectional curvature of $\operatorname{int}(Z)$ is bounded below by -1 . Let $Y_{\text {geod }}$ be a compact convex hyperbolic n-manifold with totally geodesic boundary. Let $f:(Z, \partial Z) \rightarrow\left(Y_{\text {geod }}, \partial Y_{\text {geod }}\right)$ be a homotopy equivalence of pairs. Then

$$
\operatorname{Vol}(Z) \geq \operatorname{Vol}\left(Y_{\text {geod }}\right),
$$

with equality if and only if $f$ is homotopic to an isometry.

This paper's method of proof may be of independent interest. Following [BCG1], these results are proven by defining a natural map from a nice path metric space $Z$ to a hyperbolic manifold $M_{\text {hyp }}$. Instead of obtaining this map as a uniform limit of approximating maps, the natural map is here obtained in a single step. The idea is to emulate the "short" proof of the Besson-Courtois-Gallot theorem found in [BCG2], where it is additionally assumed that $Z$ is nonpositively curved. Here the assumption of nonpositive curvature is removed, but the gist of the "short" proof is retained. As a cost for this generalization, the arguments here require that $Z$ and $M_{\text {hyp }}$ be homotopy equivalent. In [BCG1], only a map $Z \rightarrow M_{\text {hyp }}$ of nonzero degree is required.

The results in this paper represent a large portion of the author's Ph.D. thesis, completed at the University of Michigan. The author thanks his advisor, Richard Canary, for his essential assistance at every stage of this project. The author also enjoyed several helpful conversations with Yair Minsky. Thanks to Ralf Spatzier for introducing the author to the work of Besson-Courtois-Gallot.

1.1. Sketch of the proof of the main theorem. Let $Z$ be a compact $n$-dimensional convex Riemannian amalgam with universal cover $X$ (e.g. a cone-manifold or the double of a convex core), $M_{\text {hyp }}$ a closed hyperbolic $n$-manifold, and $f: Z \rightarrow M_{\text {hyp }}$ a homotopy equivalence. Up to rescaling the metric of $Z$, way may assume that $h(X)=(n-1)=h\left(\mathbb{H}^{n}\right)$. The first goal is to find a volume decreasing map $F: Z \rightarrow M_{\text {hyp }}$ homotopic to $f$. The second goal is to show that if the volume decreasing map $F$ is in fact volume preserving, then it is an isometry.

Step 1. Defining "visual measures" on $\partial X$ (Section 3). Following [BM], we define a generalization of Patterson-Sullivan measure $\left\{\mu_{x}\right\}_{x \in X}$ supported on a function theoretic compactification $\mathscr{H} X$ of $X$ by Busemann functions. As $Z$ and $M_{\text {hyp }}$ are homotopy equivalent, $X$ must be Gromov hyperbolic with Gromov boundary $\partial X$. We define an $\operatorname{Isom}(X)$-equivariant continuous surjection $\pi: \mathscr{H} X \rightarrow \partial X$, and use it to push forward the measures $\left\{\mu_{x}\right\}$ onto $\partial X$. The resulting family of probability measures $\left\{\pi_{*} \mu_{x}\right\}$ are the "visual measures" used to define the natural map $F$.

Step 2. Defining $F$ (Section 4). The homotopy equivalence $f$ lifts to a quasi-isometry between the universal covers $f: X \rightarrow \mathbb{H}^{n}$, which in turn induces a homeomorphism 
of Gromov boundaries $f: \partial X \rightarrow \partial \mathbb{H}^{n}$. The measure $\pi_{*} \mu_{x}$ is pushed forward via the boundary homeomorphism to obtain a measure $f_{*} \pi_{*} \mu_{x}$ on $\partial \mathbb{H}^{n}$. Finally, $F(x) \in \mathbb{H}^{n}$ is defined to be the barycenter of the measure $f_{*} \pi_{*} \mu_{x}$. In sum,

$$
\begin{aligned}
F: X & \rightarrow \mathbb{H}^{n}, \\
x & \mapsto \text { barycenter of measure }(f \circ \pi)_{*} \mu_{x} .
\end{aligned}
$$

It is relatively easy to show that $F$ descends to a map $F: Z \rightarrow M_{\text {hyp }}$, and is homotopic

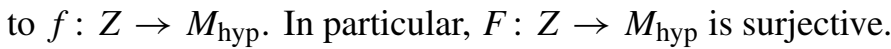

Step 3. F is locally Lipschitz (Section 5). In order to use calculus to study the map $F$, we must prove it is locally Lipschitz. (For example, a locally Lipschitz map which is infinitesimally volume decreasing almost everywhere must be volume decreasing.) This is done by factoring $F$ as a composition $F=P \circ \Phi$, such that $P$ and $\Phi$ can be analyzed directly. $\Phi: X \rightarrow L_{+}^{2}(\mathscr{H} X) \subset L^{2}(\mathscr{H} X)$ equivariantly maps $X$ into the strictly positive functions of the Hilbert space $L^{2}(\mathscr{H} X) . P: L_{+}^{2}(\mathscr{H} X) \rightarrow \mathbb{H}^{n}$ is basically the barycenter map, thinking of $L_{+}^{2}(\mathscr{H} X)$ as a space of measures. We show $\Phi$ is locally Lipschitz by direct estimates. Applying the implicit function theorem shows $P$ is $\mathcal{C}^{1}$. Together this shows $F=P \circ \Phi$ is locally Lipschitz.

Step 4. $F$ is infinitesimally volume decreasing a.e. (Section 6). With only minor modifications, the arguments of [BCG2] can be applied to show $|\mathrm{Jac} F| \leq 1$ almost everywhere. This accomplishes the first goal of showing $F$ is volume decreasing. The arguments of [BCG2] also show that if $|\operatorname{Jac} F(x)|=1$ for some $x$, then $d F_{x}$ is an infinitesimal isometry. Thus if $F$ is volume preserving, it must be an infinitesimal isometry almost everywhere.

Step 5. F is volume preserving implies it is an isometry (Section 7). Applying the arguments from [BCG1, p .790-793], we show a volume preserving map $F$ is a local isometry on an open dense set. We show $F$ is injective by using some local properties of convex Riemannian amalgams. Thus $F$ is a homeomorphism. Again using the convex Riemannian structure on $Z$, we prove $F$ is an isometry. This accomplishes the second goal, and completes the proof of Theorem 4.1.

1.2. Sketch of the applications. With the above machinery established, the theorems concerning cone-manifolds (Section 8.2), Alexandrov spaces (Section 8.3), and manifolds with boundary (Section 8.4 ) are easy to prove. To apply the machinery to hyperbolic convex cores requires more work (Section 8.1).

Recall the hypotheses of Theorem 8.1. Let $M$ and $M_{g}$ be homotopy equivalent acylindrical convex cocompact hyperbolic 3-manifolds. Assume the convex core $C_{M_{g}} \subset M_{g}$ has totally geodesic boundary. The goal is to prove $\operatorname{Vol}\left(C_{M}\right) \geq \operatorname{Vol}\left(C_{M_{g}}\right)$, with equality if and only if $M$ and $M_{g}$ are isometric. To begin, metrically double the convex cores across their boundaries to obtain the convex Riemannian amalgams $D C_{M}$ and $D C_{M_{g}}$. Notice that $D C_{M_{g}}$ is in fact a closed hyperbolic 3-manifold. A 
short argument proves there exists a $\pi_{1}$-equivariant quasi-isometric homeomorphism $f: \widetilde{D C_{M}} \rightarrow \widetilde{D C_{M_{g}}}$. Applying the above machinery to $f$ yields Theorem 8.1.

In the case where $M$ is geometrically finite with at least one rank one cusp, geometric (but not hyperbolic) Dehn surgery arguments are used to reduce to the case of closed (non-hyperbolic) manifolds, where Theorem 4.1 can be applied. (These geometric Dehn surgery techniques are based on [Bes], [L].)

\section{Preliminaries}

The following is a review of the necessary definitions. Throughout this paper, metric spaces are assumed to be complete unless otherwise stated.

2.1. $\delta$-hyperbolicity. This paper will follow the definitions and notation of [GH]. For convenience, we recall a few basic notions. Let $(X, d)$ be a $\delta$-hyperbolic space with basepoint $o \in X$. Then for $x, y \in X$, the Gromov product of $x$ and $y$ is

$$
(x \mid y):=\frac{1}{2}\{d(x, o)+d(y, o)-d(x, y)\} .
$$

A defining property of $\delta$-hyperbolic spaces is that for any triple $x, y, z \in X$,

$$
(x \mid y) \geq \min \{(x \mid z),(z \mid y)\}-\delta .
$$

The geometric content of the Gromov product may be difficult to grasp initially. The idea is that geodesic triangles in a $\delta$-hyperbolic space are very close to being tripods. For a tripod the Gromov product has the simple interpretation shown in Figure 1: it is the length of the tripod's " $o$ " leg. Since long geodesic triangles in a $\delta$-hyperbolic

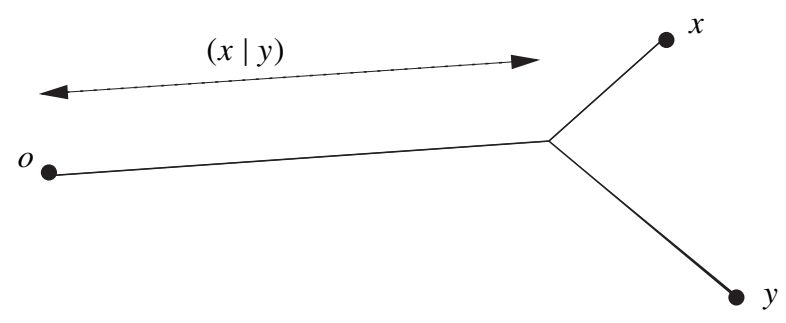

Figure 1

space are very close to tripods, on a sufficiently large scale the Gromov product is the length of the " $o$ " leg of the geodesic triangle formed by $o, x$, and $y$. 
The Gromov boundary at infinity of $X$ will be denoted by $\partial X$. The Gromov product can be extended to all of $X \cup \partial X$ as follows. For $a, b \in X \cup \partial X$, define

$$
(a \mid b):=\sup \liminf _{i, j \rightarrow \infty}\left(x_{i} \mid y_{j}\right)
$$

where the supremum is taken over all sequences $\left\{x_{i}\right\},\left\{y_{j}\right\} \subset X$ such that $x_{i} \rightarrow a$ and $y_{j} \rightarrow b$. For $a, b \in X$ this reduces to the previous definition. Using inequality (1), one can show that for any given sequences $x_{i} \rightarrow a, y_{j} \rightarrow b$,

$$
(a \mid b)-2 \delta \leq \liminf \left(x_{i} \mid y_{j}\right) \leq(a \mid b)
$$

(see [GH, p. 122]). Therefore a sequence $\left\{z_{i}\right\} \subset X \cup \partial X$ converges to $a \in \partial X$ if and only if $\left(z_{i} \mid a\right) \rightarrow \infty$.

2.2. Barycenter. Consider $\mathbb{H}^{n}$ with basepoint $o$. For each $\theta \in \partial \mathbb{H}^{n}$ let $B_{\theta}^{o}$ be the unique Busemann function of $\theta$ such that $B_{\theta}^{o}(o)=0$. Namely, if $\gamma$ is the geodesic ray based at $o$ asymptotic to $\theta$, then $B_{\theta}^{o}(p)=\lim _{t \rightarrow \infty}[d(p, \gamma(t))-t]$. Let $\lambda$ be a Radon measure on the compact space $\partial \mathbb{H}^{n}$. Define the average

$$
B_{\lambda}(p):=\int_{\partial \mathbb{H}^{n}} B_{\theta}^{o}(p) d \lambda(\theta) .
$$

Proposition 2.1 ([BCG1, Appendix A]). If $\lambda$ has no atoms, then $B_{\lambda}$ is proper and has a unique critical point in $\mathbb{H}^{n}$ corresponding to the unique global minimum. Moreover, the Hessian of $B_{\lambda}$ is a positive definite bilinear form. Namely, for all $v \in T_{p} \mathbb{H}^{n}$,

$$
\left(\text { Hess } B_{\lambda}\right)(v, v):=\left\langle\nabla_{v} \nabla B_{\lambda}, v\right\rangle>0 .
$$

The unique critical point of $B_{\lambda}$ is the barycenter of $\lambda$, denoted bar $\lambda$. Since the barycenter is the unique critical point of $B_{\lambda}$, it is defined implicitly as the unique point $p$ such that

$$
\int_{\partial \mathbb{H}^{n}}\left\langle\nabla B_{\theta}^{o}, v\right\rangle_{p} d \lambda(\theta)=0
$$

for all $v \in T_{p} \mathbb{H}^{n}$. Notice that the barycenter map is scale invariant, i.e. the barycenter of the measure $\lambda$ equals the barycenter of the measure $c \lambda$ for any $c>0$.

2.3. The brain in a jar lemma. At a crucial stage in the proof of Proposition 4.3, the following linear algebra lemma is needed.

Lemma 2.2. Let $H$ be an $n \times n$ positive definite symmetric matrix with trace $\operatorname{tr}(H)=1$. If $n \geq 3$, then

$$
\frac{\operatorname{det}(H)}{[\operatorname{det}(\operatorname{Id}-H)]^{2}} \leq\left[\frac{n}{(n-1)^{2}}\right]^{n} .
$$

Moreover, equality holds if and only if $H=\frac{1}{n} \mathrm{Id}$. 
This lemma is false for $n=2$. It is the only part of this paper (and BessonCourtois-Gallot theory in general) which fails in 2 dimensions.

2.4. Generalized differentiable and Riemannian structures ([OS]). Let $X$ be a topological space, $\Omega \subseteq X$, and $n \in \mathbb{N}$. A family $\left\{\left(U_{\phi}, \phi\right)\right\}_{\phi \in \Phi}$ is called a $\mathcal{C}^{1}$-atlas on $\Omega \subseteq X$ if the following hold:

(1) For each $\phi \in \Phi, U_{\phi}$ is an open subset of $X$.

(2) Each $\phi \in \Phi$ is a homeomorphism from $U_{\phi}$ into an open subset of $\mathbb{R}^{n}$.

(3) $\left\{U_{\phi}\right\}_{\phi \in \Phi}$ is a covering of $\Omega$.

(4) If two maps $\phi, \psi \in \Phi$ satisfy $U_{\phi} \cap U_{\psi} \neq \emptyset$, then

$$
\psi \circ \phi^{-1}: \phi\left(U_{\phi} \cap U_{\psi}\right) \rightarrow \psi\left(U_{\phi} \cap U_{\psi}\right)
$$

is $\mathcal{C}^{1}$ on $\phi\left(U_{\phi} \cap U_{\psi} \cap \Omega\right)$.

A family $\left\{g_{\phi}\right\}_{\phi \in \Phi}$ is called a $\mathcal{C}^{0}$-Riemannian metric associated with a $\mathcal{C}^{1}$-atlas $\left\{\left(U_{\phi}, \phi\right)\right\}_{\phi \in \Phi}$ on $\Omega \subseteq X$ if the following hold:

(1) For each $\phi \in \Phi, g_{\phi}$ is a map from $U_{\phi}$ to the set of positive symmetric matrices.

(2) For each $\phi \in \Phi, g_{\phi} \circ \phi^{-1}$ is continuous on $\phi\left(U_{\phi} \cap \Omega\right)$.

(3) For any $x \in U_{\phi} \cap U_{\psi}, \phi, \psi \in \Phi$, we have

$$
g_{\psi}(x)=\left[d\left(\phi \circ \psi^{-1}\right)(\psi(x))\right]^{t} g_{\phi}(x)\left[d\left(\phi \circ \psi^{-1}\right)(\psi(x))\right] .
$$

These two structures induce a distance metric $D_{g}$ on $\Omega$ which we now describe. The length of a piecewise $C^{1}$ path $\gamma:(0,1) \rightarrow \Omega$ is defined in the usual way by pulling back the metric tensor via $\gamma$. A path $\eta:[0,1] \rightarrow X$ is admissible if $\eta^{-1}(X \backslash \Omega)$ is a finite set of points $\left\{t_{1}, \ldots, t_{l}\right\}$, and $\eta$ is piecewise $\mathcal{C}^{1}$ on $(0,1) \backslash$ $\left\{t_{1}, \ldots, t_{l}\right\}$. For $x, y \in \Omega$, define

$$
D_{g}(x, y):=\inf \{\operatorname{length}(\gamma) \mid \gamma \text { joins } x \text { to } y \text { and is admissible }\} .
$$

If $x$ and $y$ cannot be joined by an admissible path, set $D_{g}(x, y)=\infty$. In general, the topology of $\left(\Omega, D_{g}\right)$ can be quite different from the subspace topology of $\Omega \subseteq X$.

2.5. Almost everywhere Riemannian spaces. Let $(X, d)$ be a geodesic metric space with Hausdorff dimension $n<\infty$. $X$ is an almost everywhere Riemannian metric space if there exists $\Omega \subseteq X$, a dense subset of full $n$-dimensional Hausdorff measure, such that:

(1) $\Omega$ admits a $n$-dimensional $\mathcal{C}^{1}$-atlas $\left\{\left(U_{\phi}, \phi\right)\right\}_{\phi \in \Phi}$.

(2) $\Omega$ admits a $\mathcal{C}^{0}$-Riemannian metric $\left\{g_{\phi}\right\}_{\phi \in \Phi}$. 
(3) Each homeomorphism $\phi \in \Phi$ is in fact locally bilipschitz.

(4) The identity map $\left(\Omega, D_{g}\right) \rightarrow(\Omega, d)$ is an isometry (see Section 2.4).

(5) The Riemannian metric induces a volume element $d \operatorname{vol}_{\Omega}$ on $\Omega$. The measure on $X$ obtained by integrating this element equals $n$-dimensional Hausdorff measure on $X(X \backslash \Omega$ has zero measure).

Notice $X$ is not assumed to be a topological manifold, and $\Omega \subseteq X$ is not assumed to be open. Conditions (1)-(5) are not as restrictive as they initially appear. For example, Otsu and Shioya proved that a finite dimensional Alexandrov space with curvature bounded below by $k \in \mathbb{R}$ is almost everywhere Riemannian [OS] (see Section 2.13). Condition (3) allows Rademacher's theorem to be applied to almost everywhere Riemannian spaces. This means locally Lipschitz functions on an almost everywhere Riemannian metric space are differentiable almost everywhere. Condition (4) says that the $\mathcal{C}^{0}$-Riemannian metric on $\Omega$ reproduces the metric $d$, in the sense that the metric completion of $\left(\Omega, D_{g}\right)$ is isometric to $(X, d)$.

2.6. Cone-manifolds ([CHK, p. 53]). An $n$-dimensional cone-manifold $M$ is a manifold which can be triangulated so that the link of each simplex is piecewise linear homeomorphic to a standard sphere and $M$ is equipped with a path metric such that the restriction of the metric to each simplex is isometric to a geodesic simplex of constant curvature $K$. The singular locus $\Sigma$ consists of the points with no neighborhood isometric to a ball in a Riemannian manifold.

It follows that

- $\Sigma$ is a union of totally geodesic closed simplices of dimension $n-2$.

- At each point of $\Sigma$ in an open $(n-2)$-simplex, there is a cone angle which is the sum of dihedral angles of $n$-simplices containing the point.

In particular, the singular locus of a 3-dimensional cone-manifold forms a graph in the manifold. A cone-manifold is an almost everywhere Riemannian metric space (see Lemma 2.3). Though a definition will not be given here, a cubed-manifold is another example of a cone-manifold (see [AMR]). More abstractly, any manifold admitting a locally finite decomposition into convex geodesic polyhedra is a cone-manifold. This can be seen by adding superfluous faces to the polyhedral decomposition.

The manifold structure of a cone-manifold will not be used in this paper. Theorem 8.6 is equally valid for more general simplicial metric spaces not satisfying the above link condition.

2.7. Convex Riemannian manifolds with boundary. A geodesic metric space $C$ is an $n$-dimensional convex Riemannian (resp. hyperbolic) manifold with boundary if

(1) $C$ is topologically an $n$-manifold with boundary,

(2) there is an incomplete Riemannian (resp. hyperbolic) metric on the interior of $C$, 
(3) the metric on $C$ is the metric completion of the Riemannian (resp. hyperbolic) metric on the interior,

(4) for any pair of points in the interior of $C$, the shortest path between them lies in the interior of $C$, and

(5) for any compact $K \subseteq C$, the curvature of the Riemannian manifold $K \cap \operatorname{int}(C)$ is bounded from above and below by finite constants.

(Notice no differentiability assumptions have been made on the boundary.)

Property (5) ensures $C$ has "locally bounded geometry". The lower curvature bound on compact sets guarantees that $C$ is locally an Alexandrov space with curvature bounded below. This implies the boundary $\partial C$ has Hausdorff dimension $n-1$ [OS]. (In [OS], they prove the singular set of an $n$-dimensional Alexandrov space with curvature bounded below has Hausdorff dimension $\leq n-1$. In this case, the boundary is the singular set.) The local upper curvature bound gives a lower bound on the volume of small metric balls in $C$. This useful property will be used in Section 7 .

Given a convex Riemannian manifold with boundary $C$, we can metrically double it across its boundary to obtain a metric space $D C$. Topologically $D C$ is the closed manifold obtained by doubling $C$ across its boundary. The metric on $D C$ is the path metric induced by gluing the two copies of $C$ (one with opposite orientation) along $\partial C$. The convexity of $C$ (property (4)) insures that the path metric obtained after doubling does not alter the original metric on $C$. Notice that $D C$ is an almost everywhere Riemannian metric space (see Lemma 2.3).

2.8. Convex Riemannian amalgams. A geodesic metric space $Z$ is an $n$-dimensional convex Riemannian amalgam if $Z$ contains an isometrically embedded locally finite countable collection $\left\{C_{j}\right\} \subseteq Z$ of $n$-dimensional convex Riemannian manifolds with boundary such that

(1) $\bigcup_{j} C_{j}=Z$,

(2) $\operatorname{int}\left(C_{j}\right) \cap \operatorname{int}\left(C_{k}\right)=\emptyset$ for $j \neq k$.

(Notice $Z$ is not assumed to be a manifold.) A cone-manifold is a convex Riemannian amalgam (see Section 2.6). Another convex Riemannian amalgam is the metric doubling $D C$ of a convex Riemannian manifold with boundary $C$ (see Section 2.7).

Lemma 2.3. A convex Riemannian amalgam is an almost everywhere Riemannian metric space.

Proof. Define $\Omega:=\bigcup_{j}$ int $\left(C_{j}\right)$. We must check conditions (1)-(5) of Section 2.5. (1)-(3) are trivial. For (4) use the following consequence of convexity: any $x, y \in Z$ can be joined by a path $\gamma$ such that

- the length of $\gamma$ is arbitrarily close to $d(x, y)$, and

- $\gamma \cap \partial C_{j}$ is at most two points for any $C_{j}$ in the decomposition. 
For (5) use that $\bigcup_{j} \partial C_{j}$ has measure zero.

Convex Riemannian amalgams seem to be the most natural class of metric spaces for which the arguments of Theorem 7.1 are valid.

2.9. Convex cores. Let $M$ be a hyperbolic manifold. Let $S \subseteq M$ be the union of all closed geodesics in $M$. The convex core, $C_{M}$, is the smallest closed convex subset of $M$ which contains $S$, in other words it is the closed convex hull of $S$ in $M$. The convex core may also be defined as the smallest closed convex subset of $M$ such that the inclusion map is a homotopy equivalence. $M$ is geometrically finite if an $\varepsilon$-neighborhood of $C_{M}$ has finite volume. Otherwise, $M$ is geometrically infinite.

For finite volume hyperbolic manifolds, the convex core is the entire manifold. Thus this is a useful object only in the infinite volume case, where $C_{M}$ is the smallest submanifold which carries all the geometry of $M$.

2.10. Pared 3-manifolds [M, Def.4.8]. Let $N$ be a compact orientable irreducible 3-manifold with nonempty boundary. Assume $N$ is not a 3-ball. Let $P \subseteq \partial N$. $(N, P)$ is a pared 3-manifold if the following three conditions hold.

(1) Every component of $P$ is an incompressible torus or a compact annulus.

(2) Every noncyclic abelian subgroup of $\pi_{1}(N)$ is conjugate into the fundamental group of a component of $P$.

(3) Every $\pi_{1}$-injective cylinder $C:\left(S^{1} \times I, S^{1} \times \partial I\right) \rightarrow(N, P)$ is relatively homotopic to a map $\psi$ such that $\psi\left(S^{1} \times I\right) \subseteq P$.

By Thurston's Geometrization Theorem $[\mathrm{M}],(N, P)$ is a pared 3-manifold if and only if there exists a geometrically finite hyperbolic structure on the interior of $N$ such that $C_{M} \cong N \backslash P$.

2.11. Pared acylindrical ([Th3, p. 244]). A pared 3-manifold $(N, P)$ is pared acylindrical if $\partial N \backslash P$ is incompressible and if every $\pi_{1}$-injective cylinder

$$
C:\left(S^{1} \times I, S^{1} \times \partial I\right) \rightarrow(N, \partial N \backslash P)
$$

is homotopic rel boundary to $\partial N$.

2.12. Deformation theory. Let $\mu>0$ be the Margulis constant for hyperbolic 3manifolds. Then for a hyperbolic 3-manifold $M$, the $\mu$-thin part of $M$ is a disjoint union of bounded Margulis tubes and unbounded cusps [BP]. After possibly making $\mu$ smaller, we may also assume that the intersection of $\partial C_{M}$ and the $\mu$-thin part of $M$ is totally geodesic [M, Lemma 6.9]. Define $M^{o}$ to be $M$ minus the unbounded components of its $\mu$-thin part. In other words, $M^{o}$ is the manifold with boundary obtained by removing the cusps from $M$. 
Let $(N, P)$ be a compact pared 3-manifold. Define the deformation space $\mathrm{H}(N, P)$ as follows. For a hyperbolic 3-manifold $M$ and a map $m: N \rightarrow M^{o},(M, m) \in$ $\mathrm{H}(N, P)$ if there exists a union $Q_{M}$ of components of $\partial M^{o}$ such that $m:(N, P) \rightarrow$ $\left(M, Q_{M}\right)$ is a relative homotopy equivalence. $\left(M_{1}, m_{1}\right)=\left(M_{2}, m_{2}\right)$ in $\mathrm{H}(N, P)$ if there exists an orientation preserving isometry $\mathcal{I}: M_{1} \rightarrow M_{2}$ such that $\mathcal{I} \circ m_{1} \sim m_{2}$. $(\mathrm{H}(N, P)$ admits several interesting topologies [Th3]. They will not be needed here. $)$ $(M, m)$ has no additional parabolics if $Q_{M}=\partial M^{o}$. Using the product structure on the complement of $C_{M}[\mathrm{EM}]$ and the thick-thin decomposition, there exists a relative homotopy equivalence $p:\left(M, Q_{M}\right) \rightarrow\left(C_{M} \cap M^{o}, C_{M} \cap Q_{M}\right)$.

Theorem 2.4 (Johannson). Let $(N, P)$ be a compact pared acylindrical 3-manifold. If $(M, m) \in \mathrm{H}(N, P)$ is geometrically finite then $p \circ m$ is homotopic to a homeomorphism $(N, P) \rightarrow\left(C_{M} \cap M^{o}, C_{M} \cap Q_{M}\right)$.

Proof. Since $N$ is not homotopy equivalent to a surface, $C_{M}$ is a 3-manifold. Since $M$ is geometrically finite, $\left(C_{M} \cap M^{o}, C_{M} \cap Q_{M}\right)$ is a compact pared 3-manifold [M, Corollary 6.10]. So $p \circ m$ is a relative homotopy equivalence between compact pared 3-manifolds, and the domain is pared acylindrical. By the work of Johannson [J, Lemma X.23, p. 235], $p \circ m$ is homotopic (rel the paring) to a homeomorphism $(N, P) \rightarrow\left(C_{M} \cap M^{o}, C_{M} \cap Q_{M}\right)$.

We are interested in pared acylindrical 3-manifolds because of the following corollary of Thurston's Geometrization Theorem and Mostow rigidity.

Corollary 2.5 ([Th2, p. 14]). Let $(N, P)$ be a pared acylindrical 3-manifold. Then there exist exactly two spaces $(M, m),\left(M^{\mathrm{opp}}, m^{\mathrm{opp}}\right) \in H(N, P)$ such that $M$ and $M^{\mathrm{opp}}$ are geometrically finite, $M$ and $M^{\mathrm{opp}}$ have no additional parabolics, and the convex cores $C_{M}$ and $C_{M}$ opp have totally geodesic boundary. Moreover, there exists an orientation reversing isometry $\mathcal{I}: M \rightarrow M^{\mathrm{opp}}$ such that $m^{\mathrm{opp}} \sim \mathcal{I} \circ \mathrm{m}$.

2.13. Alexandrov space with curvature bounded below by $\mathbf{- 1}$. There are many equivalent definitions of Alexandrov spaces. Here we give the most common definition. (See $[\mathrm{BBI}]$ for more information.)

Let $Y$ be a path metric space. $Y$ is an Alexandrov space with curvature bounded below by -1 if about each point in $Y$ there exists a neighborhood $U$ satisfying the following comparison condition. Let $x, y, z \in U$ be distinct points, let $w$ lie on the interior of a geodesic path $x y$ connecting $x$ to $y$. Let $\tilde{x}, \tilde{y}, \tilde{z}, \tilde{w} \in \mathbb{H}^{2}$ be such that $d(x, y)=d(\tilde{x}, \tilde{y}), d(y, z)=d(\tilde{y}, \tilde{z}), d(x, z)=d(\tilde{x}, \tilde{z}), d(x, w)=d(\tilde{x}, \tilde{w})$, $d(w, y)=d(\tilde{w}, \tilde{y})$. We then require that $d(z, w) \geq d(\tilde{z}, \tilde{w})$.

This comparison condition guarantees that geodesic triangles in $Y$ are at least as fat as hyperbolic triangles. The dimension of an Alexandrov space with curvature bounded below is defined to be its Hausdorff dimension. For finite dimensional 

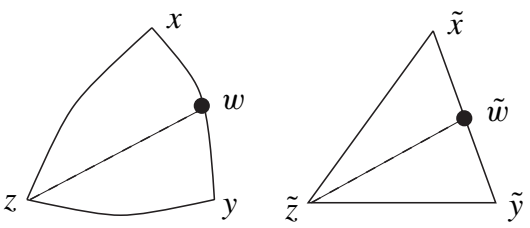

Figure 2

Alexandrov spaces with curvature bounded below, the Hausdorff and topological dimensions agree [BGP, p. 21].

In Section 8.3, we will use

Theorem 2.6 ([OS]). If $Y$ is a finite dimensional Alexandrov space with curvature bounded below by -1 , then $Y$ is an almost everywhere Riemannian metric space.

Proof. Following [OS], let $S$ be the singular set of $Y$. Define $\Omega:=Y \backslash S$. $\Omega$ is a countable intersection of dense open sets with full measure. It is therefore dense and has full measure. Conditions (1)-(5) of Section 2.5 also follow from results in [OS]. Specifically, conditions (1) and (2) follow from [Theorem B, p .630], condition (3) follows from [Lemma 5.1.3, p. 651], condition (4) follows from [Theorem B, p. 630] and [Theorem 6.4, p. 654], and condition (5) follows from [Section 7.1].

The main property of these Alexandrov spaces we will use is an upper bound on their volume growth entropy, which we now define.

2.14. Volume growth entropy. Let $X$ be a geodesic metric space of Hausdorff dimension $n, \widetilde{X}$ be the universal cover of $X$, and $\mathscr{H}^{n}$ be $n$-dimensional Hausdorff measure. The volume growth entropy of $\widetilde{X}$ is the number

$$
h(\widetilde{X}):=\limsup _{R \rightarrow \infty} \frac{1}{R} \log \mathscr{H}^{n}\left(B_{\widetilde{X}}(x, R)\right),
$$

where $x$ is any point in $\widetilde{X}$, and the ball $B_{\widetilde{X}}(x, R)$ is in $\widetilde{X}$.

The volume growth entropy is independent of the choice of $x \in \tilde{X}$.

The following theorem of Burago, Gromov, and Perelman will be important for this paper.

Theorem 2.7 ([BGP, p. 40]). If $X$ is an Alexandrov space with curvature bounded below by -1 and Hausdorff dimension $n$, then the volume growth entropy of $\widetilde{X}$ is less than or equal to the volume growth entropy of $\mathbb{H}^{n}$. In other words

$$
h(\tilde{X}) \leq h\left(\mathbb{H}^{n}\right)=n-1 .
$$




\section{Horoboundary and densities}

In order to define a density in the necessary generality, a second definition of the boundary at infinity must be made. This is a more general definition than of the Gromov boundary at infinity; it makes sense for any proper metric space. In the case of negatively curved Riemannian manifolds, it reduces to the Gromov boundary. (For more information, see [BGS, p. 21], [BM], and [F, Section 2].)

Let $Y$ be a proper metric space. For $p \in Y$, define $d_{p}(y):=d(p, y)$. Denote the space of continuous (real valued) functions on $Y$ by $C(Y)$, and endow this set with the topology of uniform convergence on compact sets. Define an equivalence relation on $C(Y)$ by $f \sim g$ if and only if $f-g$ is a constant function. Denote the quotient space $C(Y) / \sim$ by $C_{*}(Y)$. $C_{*}(Y)$ is Hausdorff. Define a map $\iota: Y \rightarrow C_{*}(Y)$ by $\iota(p):=\left[d_{p}\right] . \iota$ is a topological embedding.

Definition 3.1. Let $\mathrm{Cl}(Y)$ denote the closure of $\iota(Y)$ in $C_{*}(Y)$. The horoboundary of $Y$ is

$$
\mathscr{H} Y:=\mathrm{Cl}(Y) \backslash \iota(Y) .
$$

A continuous function $h \in C(Y)$ such that $[h] \in \mathscr{H} Y$ is a horofunction of $Y$.

For $\eta \in C_{*}(Y)$ define a function $b_{\eta}: Y \times Y \rightarrow \mathbb{R}$ by

$$
b_{\eta}(p, q):=h(p)-h(q) \text { for any } h \in C(Y) \text { such that }[h]=\eta \text {. }
$$

If $Y$ has a fixed basepoint $o$, then define $b_{\eta}(p):=b_{\eta}(p, o)$.

It is a quick check to see that $b_{\eta}$ is well-defined, i.e. independent of the choice of $h$. The functions $b_{\eta}$ are 1-Lipschitz. Thus applying the Arzela-Ascoli theorem shows $\mathrm{Cl}(Y)$ is compact, implying $\mathscr{H} Y$ is compact. (If $Y$ is nonpositively curved, then horofunctions and the horoboundary are identical to Busemann functions and the boundary at infinity [BGS, p. 22].)

Isometries of $Y$ extend to homeomorphisms of $\mathscr{H} Y$ in the following simple manner. Consider the $\operatorname{Isom}(Y)$-action by homeomorphisms on $C(Y)$ given by $\phi . f:=$ $f \circ \phi^{-1}$. This action descends to an action on $C_{*}(Y)$. Since $\phi \cdot\left[d_{p}\right]=\left[d_{\phi . p}\right]$, the map $\left.\phi\right|_{\iota(Y)}: \iota(Y) \rightarrow \iota(Y)$ is a homeomorphism. Since $\phi$ acts as a homeomorphism on both $\iota(Y)$ and $C_{*}(Y)$, it also acts as a homeomorphism on $\mathscr{H} Y$. Thus we have defined an $\operatorname{Isom}(Y)$-action by homeomorphisms on $\mathscr{H} Y$.

Definition 3.2. Let $Y$ be proper metric space. Let $G$ be a closed subgroup of Isom $(Y)$. A continuous map (under the weak- $*$ topology on measures)

$$
\mu: Y \rightarrow \text { ppositive Radon measures on } \mathscr{H} Y \text { \} }
$$

is an $\ell$-dimensional density for $G$ if 
(1) $\mu$ is $G$-equivariant, i.e. $\mu_{g . x}=g_{*} \mu_{x}$,

(2) $\mu_{p} \ll \mu_{q}$ for all $p, q$, and for $\eta \in \mathscr{H} Y$,

$$
\frac{d \mu_{p}}{d \mu_{q}}(\eta)=e^{-\ell\left(b_{\eta}(p, q)\right)}
$$

Example. For $\mathbb{H}^{n}$, $\mathscr{H} \mathbb{H}^{n}=\partial \mathbb{H}^{n}$. Let $\Phi: S_{p} \mathbb{H}^{n} \rightarrow \partial \mathbb{H}^{n}$ be the standard radial homeomorphism between the unit tangent sphere and the boundary at infinity. Define $\mu_{p}$ to be the push-forward of Lebesgue measure on $S_{p} Y$ by $\Phi$. This is known as the visual measure at $p$, and is an $(n-1)$-dimensional density for all of Isom $\left(\mathbb{H}^{n}\right)$. Visual measure is the most natural density in this case.

Our entire reason for defining the horoboundary is the following proposition.

Proposition 3.1 ([BM, Prop. 1.1]). Let $X$ be a proper metric space of Hausdorff dimension $n$ with basepoint $o \in X$. Let $m$ be $n$-dimensional Hausdorff measure. Assume $m(X)=\infty$ and $X$ has finite volume growth entropy $h(X)$. Then there exists an $h(X)$-dimensional density $x \mapsto \mu_{x}$ for $\operatorname{Isom}(X)$. This density is called Patterson-Sullivan measure.

By normalizing we may always assume that $\mu_{o}$ is a probability measure. Notice this normalization implies that $\mu_{g . o}=g_{*} \mu_{o}$ is also a probability measure for any $g \in \operatorname{Isom}(X)$.

Let $X$ be a $\delta$-hyperbolic proper path metric space. Assume that $h(X) \in(0, \infty)$. We have defined two different compactifications of $X$; the Gromov boundary at infinity $\partial X$ and the horoboundary $\mathscr{H} X$. In general, those two compactifications are not homeomorphic. However, they are both necessary for the work of this paper. To connect the two compactifications, we now define a continuous $\operatorname{Isom}(X)$-equivariant surjection $\pi: \mathscr{H} X \rightarrow \partial X$.

Fix a basepoint $o \in X$. Pick $\xi \in \mathscr{H} X$ and a sequence $\left\{p_{n}\right\}$ such that $\left[d_{p_{n}}\right] \rightarrow \xi$ in $C_{*}(X)$. Then $\left\{p_{n}\right\}$ leaves every compact set of $X$. So by the Arzela-Ascoli theorem there exists a subsequence $\left\{a_{l}\right\} \subseteq\left\{p_{n}\right\}$ such that the geodesic segments $o a_{l}$ converge to some $[\gamma] \in \partial X$ in the compact-open topology, where $\gamma$ is a geodesic ray based at $o$.

Define $\pi: \mathscr{H} X \rightarrow \partial X$ by $\pi(\xi)=[\gamma]$. Before proving $\pi$ is well defined, we need the following lemma.

Lemma 3.2. In the above notation, the sequence $\left\{a_{l}\right\}$ converges to $[\gamma] \in \partial X$.

Proof. It is enough to show $\left([\gamma] \mid a_{l}\right) \rightarrow \infty$. Pick a large $M \gg 0$, and consider the metric ball $B(o, M)$. Find $L$ such that for $l \geq L$, the geodesic segment $o a_{l}$ is 1-close to $\gamma$ on $B(o, M)$. Then the picture looks roughly like Figure 3 . 


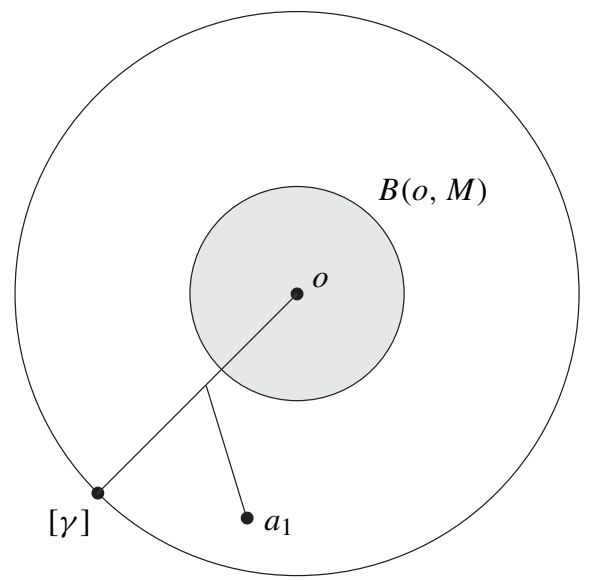

Figure 3

As $M \rightarrow \infty$, intuitively $\left([\gamma] \mid a_{l}\right) \rightarrow \infty$. To prove this carefully, notice that for $k>1$

$$
d\left(\gamma(k M), a_{l}\right) \leq\left[d\left(a_{l}, o\right)-M\right]+[d(\gamma(k M), o)-M]+1 .
$$

This implies

$$
\left(\gamma(k M) \mid a_{l}\right) \geq M-1 / 2 .
$$

So finally

$$
\left([\gamma] \mid a_{l}\right) \geq \liminf _{k \rightarrow \infty}\left(\gamma(k M) \mid a_{l}\right)-2 \delta \geq M-2 \delta-1 / 2 .
$$

Lemma 3.3. The map $\pi: \mathscr{H} X \rightarrow \partial X$ is well defined.

Proof. Suppose $\pi$ is not well defined. Then there exist sequences $p_{i}, q_{i} \rightarrow \xi \in \mathscr{H} X$ such that $\left\{p_{i}\right\},\left\{q_{i}\right\}$ do not converge to a common point in $\partial X$. Thus there exists an $M>0$ such that $\left(p_{i} \mid q_{i}\right)<M$ for all $i$.

Pick a metric closed ball $K$ much larger than $B(o, M) . p_{i}, q_{i} \rightarrow \xi \in \mathscr{H} X$ implies that for large $i$ and all $x \in K$,

$$
d\left(p_{i}, x\right)-d\left(p_{i}, o\right) \approx d\left(q_{i}, x\right)-d\left(q_{i}, o\right) .
$$

This will lead to a contradiction.

To begin, pick $x \in K \cap o q_{i}$ such that $d(x, o) \gg M$. Then

$$
d\left(q_{i}, x\right)-d\left(q_{i}, o\right)=-d(x, o) .
$$




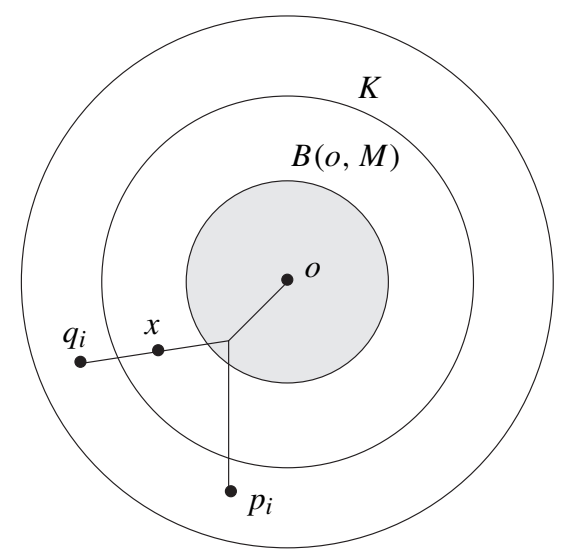

Figure 4

Approximate the four point metric space $\left\{x, o, q_{i}, p_{i}\right\}$ by a tree. Let the corresponding four points in the tree be $\left\{\tilde{x}, \tilde{o}, \tilde{q}_{i}, \tilde{p}_{i}\right\}$. We may assume this tree is at worst $(1,2 \delta)$-quasi-isometric to $\left\{x, o, q_{i}, p_{i}\right\}[\mathrm{GH}, \mathrm{p} .33]$ (see Figure 4). We thus obtain the inequalities

$$
\begin{aligned}
d\left(p_{i}, x\right)-d\left(p_{i}, o\right) & \geq d\left(\tilde{p}_{i}, \tilde{x}\right)-d\left(\tilde{p}_{i}, \tilde{o}\right)-4 \delta \\
& =d(\tilde{x}, \tilde{o})-2\left(\tilde{x} \mid \tilde{p}_{i}\right)-4 \delta \\
& =d(\tilde{x}, \tilde{o})-2\left(\tilde{q}_{i} \mid \tilde{p}_{i}\right)-4 \delta \\
& \geq d(x, o)-2 \delta-2\left(q_{i} \mid p_{i}\right)-6 \delta-4 \delta \\
& \geq d(x, o)-2 M-12 \delta>\frac{d(x, o)}{2} .
\end{aligned}
$$

Together with equation (3), this contradicts equation (2).

Lemma 3.4. $\pi$ is continuous and surjective.

Proof. To see that $\pi$ is surjective, pick a geodesic ray $\gamma$ based at $o$. By compactness there is a sequence $\left\{t_{i}\right\} \subset(0, \infty)$ such that $\gamma\left(t_{i}\right)$ converges to a point $\xi \in \mathscr{H} X$. Then by definition $\pi(\xi)=[\gamma]$.

Let $\xi^{n} \rightarrow \xi$ in $\mathscr{H} X$. Pick sequences $\left\{p_{i}^{n}\right\}$ such that for all $n, \lim _{i \rightarrow \infty} p_{i}^{n}=\xi^{n}$. This implies $\lim _{i \rightarrow \infty} p_{i}^{n}=\pi\left(\xi^{n}\right)$ in $\partial X$ for all $n$. There is an increasing sequence $i_{1}, i_{2}, i_{3}, \ldots$ of natural numbers such that

$$
\lim _{n \rightarrow \infty} p_{i_{n}}^{n}=\xi \text { in } \mathscr{H} X, \quad \text { and } \quad\left(\pi\left(\xi^{n}\right) \mid p_{i_{n}}^{n}\right)>n
$$


This implies $\lim _{n \rightarrow \infty} p_{i_{n}}^{n}=\pi(\xi)$ in $\partial X$. By the definition of $\delta$-hyperbolicity

$$
\left(\pi(\xi) \mid \pi\left(\xi^{n}\right)\right) \geq\left[\min \left\{\left(\pi(\xi) \mid p_{i_{n}}^{n}\right),\left(\pi\left(\xi^{n}\right) \mid p_{i_{n}}^{n}\right)\right\}-3 \delta\right] \rightarrow \infty \quad \text { as } n \rightarrow \infty .
$$

Therefore $\pi\left(\xi^{n}\right) \rightarrow \pi(\xi)$ in $\partial X$.

The proof of the following lemma is trivial and has been omitted.

Lemma 3.5. For all $g \in \operatorname{Isom}(X), g \circ \pi=\pi \circ g$.

Lemma 3.6. If $X$ admits a cocompact isometric action, then $\pi_{*} \mu_{p}$ has no atoms for all $p \in X$.

Proof. As $\pi_{*} \mu_{p} \ll \pi_{*} \mu_{o}$, it is sufficient to show $\pi_{*} \mu_{o}$ has no atoms. Suppose there exists $\alpha \in \partial X$ such that $\pi_{*} \mu_{o}(\alpha)>0$. Since $X$ admits a cocompact isometric action, there is a constant $D>0$ such that any $p \in X$ is at most a distance $D$ from an orbit point $g_{p} . o$ for $g_{p} \in \operatorname{Isom}(X)$. It follows that the total mass of the measure $\mu_{p}$ is at most $e^{D h(X)}$.

We first show all the horofunctions in the fiber $\pi^{-1}(\alpha)$ are a bounded distance from each other. Pick a geodesic ray in $X$ based at $o$ asymptotic to $\alpha$. Pick a sequence $p_{i}$ of points going to infinity on the geodesic ray. After passing to a subsequence we may assume the points $p_{i}$ converge to some $\xi \in \pi^{-1}(\alpha) \subset \mathscr{H} X$.

By definition

$$
b_{\xi}(x)=\lim \left(-2\left(p_{i} \mid x\right)+d(x, o)\right) .
$$

We know

$$
(\alpha \mid x)-2 \delta \leq \liminf \left(p_{i} \mid x\right) \leq(\alpha \mid x) .
$$

Because $b_{\xi}$ is well defined, the lim inf can be replaced by a limit. Thus

$$
-2(\alpha \mid x)+d(x, o) \leq b_{\xi}(x) \leq-2(\alpha \mid x)+d(x, o)+4 \delta .
$$

So for any other $\zeta \in \pi^{-1}(\alpha)$ we have

$$
\left|b_{\xi}(x)-b_{\zeta}(x)\right| \leq 4 \delta .
$$

Using this we obtain the inequality

$$
\begin{aligned}
\pi_{*} \mu_{p_{i}}(\alpha)=\int_{\pi^{-1}(\alpha)} d \mu_{p_{i}} & =\int_{\pi^{-1}(\alpha)} e^{-h(X) b_{\zeta}\left(p_{i}\right)} d \mu_{o}(\zeta) \\
& \geq \int_{\pi^{-1}(\alpha)} e^{-h(X)\left[b_{\xi}\left(p_{i}\right)+4 \delta\right]} d \mu_{o}(\zeta) \\
& =e^{-h(X)\left[b_{\xi}\left(p_{i}\right)+4 \delta\right]} \cdot \pi_{*} \mu_{o}(\alpha)>0 .
\end{aligned}
$$


But $\lim _{i \rightarrow \infty} b_{\xi}\left(p_{i}\right)=-\infty$. So

$$
\lim _{i \rightarrow \infty} \pi_{*} \mu_{p_{i}}(\alpha)=\infty
$$

Since $\pi_{*} \mu_{p_{i}}$ has total mass at most $e^{D h(X)}$, this is a contradiction. Therefore $\pi_{*} \mu_{o}$ has no atoms.

\section{The Besson-Courtois-Gallot inequality}

Theorem 4.1. Let $M_{\mathrm{hyp}}$ be a closed hyperbolic $n$-manifold for $n \geq 3$. Let $Z$ be a compact n-dimensional almost everywhere Riemannian metric space with universal cover X. (For a definition, see Section 2.5.) Let $f: Z \rightarrow M_{\text {hyp }}$ be a homotopy equivalence. Then

$$
h(X)^{n} \operatorname{Vol}(Z) \geq(n-1)^{n} \operatorname{Vol}\left(M_{\mathrm{hyp}}\right) .
$$

Let $f$ also denote the lifted map $f: X \rightarrow \mathbb{H}^{n}$. Recall that if $Z$ and $M_{\text {hyp }}$ are compact, then a homotopy equivalence $f: Z \rightarrow M_{\text {hyp }}$ lifts to a quasi-isometry $f: X \rightarrow \widetilde{M_{\text {hyp }}}$ between the universal covers. This implies that the volume growth entropy of $X$ is a strictly positive and finite (see for example [Gr, Prop.5.10]). Fix a basepoint $o \in X$, and let $f(o) \in \mathbb{H}^{n}$ be a basepoint of $\mathbb{H}^{n}$. Since $f: X \rightarrow \mathbb{H}^{n}$ is a quasi-isometry, $f$ extends to a homeomorphism between the Gromov boundaries $f: \partial X \rightarrow \partial \mathbb{H}^{n}$.

We now define the natural map $F: X \rightarrow \mathbb{H}^{n}$, but postpone proving its regularity properties until later sections. For $x \in X$, let $\mu_{x}$ be the Patterson-Sullivan measure at $x$. Recall we have defined a continuous map $\pi: \mathscr{H} X \rightarrow \partial X$. Push forward the Patterson-Sullivan measure $\mu_{x}$ on $\mathscr{H} X$ to a probability measure $(f \circ \pi)_{*} \mu_{x}$ on $\partial \mathbb{H}^{n}$. Define $F: X \rightarrow \mathbb{H}^{n}$ by

$$
F(x):=\operatorname{barycenter}\left((f \circ \pi)_{*} \mu_{x}\right) .
$$

$F$ is the natural map induced by $f . F$ is a $\Gamma:=\pi_{1}(Z) \cong \pi_{1}\left(M_{\text {hyp }}\right)$-equivariant continuous map. It therefore descends to a continuous map $F: Z \rightarrow M_{\text {hyp }}$.

Remark 4.2. Any additional symmetries of the map $f: Z \rightarrow M_{\text {hyp }}$ also become symmetries of $F$. Namely, if $Z$ and $M_{\text {hyp }}$ possess an isometric involution, and $f: Z \rightarrow M_{\text {hyp }}$ is equivariant with respect to the involutions, then $F: Z \rightarrow M_{\text {hyp }}$ is similarly equivariant. This follows immediately from the definition, because both $\pi: \mathscr{H} X \rightarrow \partial X$ and Patterson-Sullivan measure are $\operatorname{Isom}(X)$-equivariant. This fact will be used in the proofs of Theorems 8.1 and 8.9. 
$F, f: X \rightarrow \mathbb{H}^{n}$ are $\Gamma$-equivariant maps. The straight-line homotopy between them is also $\Gamma$-equivariant. Therefore the downstairs maps $F, f: Z \rightarrow M_{\text {hyp }}$ are homotopic. This shows $F: Z \rightarrow M_{\text {hyp }}$ is a homotopy equivalence. As $M_{\text {hyp }}$ is a closed manifold, $F$ must be surjective.

Proving the local regularity properties of the natural map requires some work. These properties are summarized in

Proposition 4.3. The natural map $F: Z \rightarrow M_{\text {hyp }}$ has the following properties:

(1) It is locally Lipschitz and differentiable almost everywhere.

(2) $|\operatorname{Jac} F(p)| \leq\left(\frac{h(X)}{n-1}\right)^{n}$ almost everywhere.

(3) If for some $p,|\operatorname{Jac} F(p)|=\left(\frac{h(X)}{n-1}\right)^{n}$, then the differential $d F_{p}$ is a homothety of ratio $\left(\frac{h(X)}{n-1}\right)^{n}$.

This proposition will be proven in later sections. Specifically, (1) will be proven in Section 5, (2) and (3) will be proven in Section 6. Let us now temporarily assume it, and complete the proof of Theorem 4.1. (In this section, we use only (1) and (2). (3) will not be used until Section 7.)

By assumption, $Z$ is an almost everywhere Riemannian metric space. So by definition $Z$ has a subset $\Omega$ of full measure admitting a $\mathcal{C}^{1}$-atlas $\left\{U_{\phi}, \phi\right\}_{\phi \in \Phi}$ and a $\mathcal{C}^{0}$-Riemannian metric $\left\{g_{\phi}\right\}_{\phi \in \Phi}$. This Riemannian metric induces a volume element $\omega_{Z}$ which agrees with $n$-dimensional Hausdorff measure on $Z$. Let $\omega_{M_{\text {hyp }}}$ be the volume element on $M_{\text {hyp }}$.

\section{Lemma 4.4.}

$$
\operatorname{Vol}\left(M_{\mathrm{hyp}}\right)=\operatorname{Vol}(F(Z))=\int_{F(Z)} \omega_{M_{\mathrm{hyp}}} \leq \int_{Z}|\operatorname{Jac} F| \omega_{Z} \leq\left(\frac{h(X)}{n-1}\right)^{n} \operatorname{Vol}(Z) .
$$

Proof. Assuming Proposition 4.3, the only non-trivial part is to prove

$$
\int_{F(Z)} \omega_{M_{\text {hyp }}} \leq \int_{Z}|\operatorname{Jac} F| \omega_{Z}
$$

This amounts to justifying the change of variables formula for the singular space $Z$. To do this, we will unpack the definitions and apply the change of variables formula for Lipschitz maps.

$Z \backslash \Omega$ is measure zero, and $F$ is locally Lipschitz. This implies $F(Z \backslash \Omega) \subset M_{\text {hyp }}$ is also measure zero. The collection of open sets $\left\{U_{\phi}\right\}_{\phi \in \Phi}$ covers $\Omega$. Let $\left\{E_{k}\right\}$ be a countable partition of $\cup_{\phi} U_{\phi} \subset Z$ into measurable sets such that each $E_{k}$ is contained in some open set $U_{\phi}$. Since $F\left(\cup_{k} E_{k}\right)=F\left(\cup_{\phi} U_{\phi}\right) \subset M_{\text {hyp }}$ is of full measure, it 
suffices to prove the inequality on each measureable set $E_{k}$, i.e. it suffices to show that

$$
\int_{F\left(E_{k}\right)} \omega_{M_{\mathrm{hyp}}} \leq \int_{E_{k}}|\mathrm{Jac} F| \omega_{Z}
$$

Assume that $E_{k} \subset U_{\phi}$. Let us also assume without a loss of generality that the image $F\left(U_{\phi}\right) \subset M_{\text {hyp }}$ lies in an open set $V_{M}$ equipped with a smooth diffeomorphism $\psi$ onto an open subset of $\mathbb{R}^{n}$. Let $g_{M}$ denote the smooth Riemannian metric on $\psi\left(V_{M}\right)$ given by the hyperbolic metric on $M_{\text {hyp }}$.

The volume element $\left(\phi^{-1}\right)^{*}\left(\left.\omega_{Z}\right|_{U_{\phi}}\right)$ is defined on an open subset of $\mathbb{R}^{n}$. It is induced by the $\mathcal{C}^{0}$-Riemannian metric $g_{\phi}$. Concretely this means that

$$
\left(\phi^{-1}\right)^{*}\left(\left.\omega_{Z}\right|_{U_{\phi}}\right)=\sqrt{\operatorname{det} g_{\phi}} d x_{1} d x_{2} \ldots d x_{n}
$$

Similarly,

$$
\left(\psi^{-1}\right)^{*} \omega_{M_{\mathrm{hyp}}}=\sqrt{\operatorname{det} g_{M}} d y_{1} d y_{2} \ldots d y_{n} .
$$

Define the locally Lipschitz map $G:=\psi \circ F \circ \phi^{-1}$, which is a map between subsets of Euclidean space. By definition, the Jacobian of $F: Z \rightarrow M_{\text {hyp }}$ at $p \in U_{\phi}$ is

$$
|\operatorname{Jac} F(p)|:=\frac{\sqrt{\operatorname{det} g_{M}(\psi(F(p)))}}{\sqrt{\operatorname{det} g_{\phi}(\phi(p))}} \cdot|\operatorname{Jac} G(\phi(p))| .
$$

Applying the change of variables formula for Lipschitz maps [EG, 3.4.3] to $G$ yields

$$
\begin{aligned}
\int_{\phi\left(U_{\phi}\right)} & \chi_{\phi\left(E_{k}\right)}(x) \sqrt{\operatorname{det} g_{M}(G(x))}|\operatorname{Jac} G(x)| d x_{1} d x_{2} \ldots d x_{n} \\
= & \int_{\psi\left(V_{M}\right)} \#\left\{G^{-1}(y) \cap \phi\left(E_{k}\right)\right\} \sqrt{\operatorname{det} g_{M}(y)} d y_{1} d y_{2} \ldots d y_{n} .
\end{aligned}
$$

Since det $g_{\phi}$ vanishes on a set of measure zero, way may perform the following step

$$
\begin{aligned}
\int_{\phi\left(U_{\phi}\right)} & \chi_{\phi\left(E_{k}\right)}(x) \sqrt{\operatorname{det} g_{M}(G(x))}|\operatorname{Jac} G(x)| d x_{1} d x_{2} \ldots d x_{n} \\
= & \int_{\phi\left(U_{\phi}\right)} \chi_{\phi\left(E_{k}\right)}(x) \frac{\sqrt{\operatorname{det} g_{M}(G(x))}}{\sqrt{\operatorname{det} g_{\phi}(x)}} \sqrt{\operatorname{det} g_{\phi}(x)}|\operatorname{Jac} G(x)| d x_{1} d x_{2} \ldots d x_{n} \\
= & \int_{\phi\left(U_{\phi}\right)} \chi_{\phi\left(E_{k}\right)}(x)\left|\operatorname{Jac} F\left(\phi^{-1}(x)\right)\right| \sqrt{\operatorname{det} g_{\phi}(x)} d x_{1} d x_{2} \ldots d x_{n} .
\end{aligned}
$$


Putting this together yields

$$
\begin{aligned}
\int_{E_{k}}|\operatorname{Jac} F(z)| \omega_{Z} & =\int_{\phi\left(U_{\phi}\right)} \chi_{\phi\left(E_{k}\right)}(x)\left|\operatorname{Jac} F\left(\phi^{-1}(x)\right)\right| \sqrt{\operatorname{det} g_{\phi}(x)} d x_{1} d x_{2} \ldots d x_{n} \\
& =\int_{\psi\left(V_{M}\right)} \#\left\{G^{-1}(y) \cap \phi\left(E_{k}\right)\right\} \sqrt{\operatorname{det} g_{M}(y)} d y_{1} d y_{2} \ldots d y_{n} \\
& =\int_{V_{M}} \#\left\{F^{-1}(m) \cap E_{k}\right\} \omega_{M_{\mathrm{hyp}}}(m) \geq \int_{F\left(E_{k}\right)} \omega_{M_{\mathrm{hyp}}} .
\end{aligned}
$$

The above lemma implies that

$$
h(X)^{n} \operatorname{Vol}(Z) \geq(n-1)^{n} \operatorname{Vol}\left(M_{\mathrm{hyp}}\right) .
$$

This proves the inequality of Theorem 4.1.

\section{The Barycenter map is locally Lipschitz}

In this section we prove part (1) of Proposition 4.3.

(1) The natural map $F: Z \rightarrow M_{\text {hyp }}$ is locally Lipschitz and differentiable almost everywhere.

It is only necessary to prove $F$ is locally Lipschitz. Almost everywhere differentiability will then follow by using Rademacher's theorem (see Section 2.5). We will prove the lifted map $F: X \rightarrow \mathbb{H}^{n}$ is locally Lipschitz by factoring it as a composition of two locally Lipschitz maps. Namely we will define a locally Lipschitz map $\Phi: X \rightarrow L^{2}(\mathscr{H} X)$ and a $\mathcal{C}^{1}$-map $P: L^{2}(\mathcal{H} X) \rightarrow \mathbb{H}^{n}$ such that $F=P \circ \Phi$.

The barycenter map takes a positive atomless measure $v$ on $\partial \mathbb{H}^{n}$ to the unique point $x=\operatorname{bar}(v)$ defined implicitly by the equation

$$
\int_{\partial \mathbb{H}^{n}}\left\langle\nabla B_{\theta}^{o}, v\right\rangle_{x} d v(\theta)=0 \quad \text { for all } v \in T_{x} \mathbb{H}^{n},
$$

where $B^{o}$ is the Busemann function on $\mathbb{H}^{n}$ (normalized so $B^{o}(o, \theta)=0$ for all $\left.\theta \in \partial \mathbb{H}^{n}\right)$.

Consider the Hilbert space $L^{2}(\mathscr{H} X)$ of square integrable functions on $\mathscr{H} X$ with respect to the Patterson-Sullivan probability measure $\mu_{o}$. Define a $\Gamma:=\pi_{1}(Z)$ action on $L^{2}(\mathscr{H} X)$ by

$$
(\gamma \cdot \phi)(\eta):=\phi\left(\gamma^{-1} . \eta\right) \cdot \sqrt{\exp \left(-h(X) b_{\eta}(\gamma \cdot o)\right)} .
$$

Lemma 5.1. $\Gamma$ acts by isometries on $L^{2}(\mathscr{H} X)$. 
Proof.

$$
\begin{aligned}
\int_{\mathscr{H} X}(\gamma \cdot \phi)^{2}(\eta) d \mu_{o}(\eta) & =\int_{\mathscr{H} X} \phi^{2}\left(\gamma^{-1} \cdot \eta\right) e^{-h(X) b_{\eta}(\gamma \cdot o)} d \mu_{o}(\eta) \\
& =\int_{\mathscr{H} X} \phi^{2}\left(\gamma^{-1} \cdot \eta\right) d \mu_{\gamma . o}(\eta) \\
& =\int_{\mathscr{H} X} \phi^{2}(\eta) d\left(\gamma_{*}^{-1} \mu_{\gamma . o}\right)(\eta)=\int_{\mathscr{H} X} \phi^{2} d \mu_{o}
\end{aligned}
$$

Let $L_{+}^{2}(\mathscr{H} X)$ denote the strictly positive functions in $L^{2}(\mathscr{H} X)$. Notice that $\Gamma$ acts by isometries on $L_{+}^{2}(\mathscr{H} X)$. An element $\phi \in L_{+}^{2}(\mathscr{H} X)$ defines a positive atomless measure $\phi^{2} d \mu_{o}$. Push this measure forward via the map $f \circ \pi$ to a measure $(f \circ \pi)_{*}\left(\phi^{2} d \mu_{o}\right)$ on $\partial \mathbb{H}^{n}$. Define the map $P: L_{+}^{2}(\mathcal{H} X) \rightarrow \mathbb{H}^{n}$ by

$$
P: \phi \mapsto \operatorname{bar}\left((f \circ \pi)_{*}\left(\phi^{2} d \mu_{o}\right)\right) .
$$

In other words $P(\phi)$ is the unique point $x$ defined implicitly by the equation

$$
\int_{\partial \mathbb{H}^{n}}\left\langle\nabla B_{\theta}^{o}, v\right\rangle_{x} d\left((f \circ \pi)_{*}\left(\phi^{2} d \mu_{o}\right)\right)(\theta)=\int_{\mathscr{H} X}\left\langle\nabla B_{f \circ \pi(\eta)}^{o}, v\right\rangle_{x} \phi^{2}(\eta) d \mu_{o}=0,
$$

for all $v \in T_{x} \mathbb{H}^{n}$.

\section{Lemma 5.2. $P$ is $\Gamma$-equivariant.}

Proof. $P(\gamma . \phi)$ is the unique point $x$ such that for all $v \in T_{x} \mathbb{H}^{n}$,

$$
\begin{aligned}
0 & =\int_{\mathscr{H} X}\left\langle\nabla B_{f \circ \pi(\eta)}^{o}, v\right\rangle_{x} \phi^{2}\left(\gamma^{-1} \eta\right) e^{-h(X) b_{\eta}(\gamma . o)} d \mu_{o}(\eta) \\
& =\int_{\mathscr{H} X}\left\langle\nabla B_{f \circ \pi(\eta)}^{o}, v\right\rangle_{x} \phi^{2}\left(\gamma^{-1} \eta\right) d \mu_{\gamma . o}(\eta) \\
& =\int_{\mathscr{H} X}\left\langle\nabla B_{f \circ \pi \circ \gamma(\eta)}^{o}, v\right\rangle_{x} \phi^{2}(\eta) d \mu_{o}(\eta) \quad \text { (change of variables) } \\
& =\int_{\mathscr{H} X}\left\langle\nabla B_{\gamma \circ f \circ \pi(\eta)}^{o}, v\right\rangle_{x} \phi^{2}(\eta) d \mu_{o}(\eta) \quad(f \circ \pi \text { is } \Gamma \text {-equivariant) } \\
& =\int_{\mathscr{H} X}\left\langle\nabla B_{f \circ \pi(\eta)}^{o}, d \gamma^{-1}(v)\right\rangle_{\gamma^{-1} . x} \phi^{2}(\eta) d \mu_{o}(\eta) \quad\left(\nabla B^{o} \text { is Isom( }\left(\mathbb{H}^{n}\right)\right. \text {-invariant). }
\end{aligned}
$$

Since $d \gamma^{-1}: T_{x} \mathbb{H}^{n} \rightarrow T_{\gamma^{-1} . x} \mathbb{H}^{n}$ is an isomorphism, this implies $\gamma^{-1} \cdot x=P(\phi)$. Therefore $P(\gamma . \phi)=x=\gamma \cdot P(\phi)$. 
Pick a $\mathcal{C}^{\infty}$ frame $\left\{e_{i}\right\}$ on $\mathbb{H}^{n}$ and define a map $Q: \mathbb{H}^{n} \times L_{+}^{2}(\mathcal{H} X) \rightarrow \mathbb{R}^{n}$ by

$Q:(x, \phi) \mapsto$

$$
\left(\int_{\mathscr{H} X}\left\langle\nabla B_{f \circ \pi(\eta)}^{o}, e_{1}\right\rangle_{x} \phi^{2}(\eta) d \mu_{o}(\eta), \ldots, \int_{\mathcal{H} X}\left\langle\nabla B_{f \circ \pi(\eta)}^{o}, e_{n}\right\rangle_{x} \phi^{2}(\eta) d \mu_{o}(\eta)\right) .
$$

$\mathscr{H} X$ is compact, $B_{\theta}^{o}(x)$ is a $\mathcal{C}^{\infty}$ function of both $\theta$ and $x$, and $\left\{e_{i}\right\}$ is a $\mathcal{C}^{\infty}$ frame. Using these facts, applying the Lebesgue dominated convergence theorem proves that $Q$ is $\mathcal{C}^{\infty}$. Notice $P$ is defined implicitly by the equation

$$
Q(P(\phi), \phi)=(0, \ldots, 0) .
$$

The goal is to show that $P$ is $\mathcal{C}^{1}$. This can be accomplished by employing the implicit function theorem. (The implicit function theorem is true on Banach spaces. See [RS, p. 366].) For each fixed $\phi$, we must show the map $Q^{\phi}: x \mapsto Q(x, \phi)$ has an invertible differential at each point $x$ of the fiber $\left(Q^{\phi}\right)^{-1}(0, \ldots, 0)$. Split $Q^{\phi}$ into coordinate functions $Q^{\phi}=\left(Q_{1}^{\phi}, \ldots, Q_{n}^{\phi}\right)$. Then

$$
\begin{aligned}
\frac{\partial}{\partial x_{j}} Q_{i}^{\phi}(x)= & \int_{\mathscr{H} X}\left(\operatorname{Hess} B_{f \circ \pi(\eta)}^{o}\right)_{x}\left(e_{j}, e_{i}\right) \phi^{2}(\eta) d \mu_{o}(\eta) \\
& +\int_{\mathscr{H} X}\left\langle\nabla B_{f \circ \pi(\eta)}^{o}, \nabla_{e_{j}} e_{i}\right\rangle_{x} \phi^{2}(\eta) d \mu_{o}(\eta) .
\end{aligned}
$$

The second term in this sum satisfies

$$
\begin{aligned}
\int_{\mathscr{H} X}\left\langle\nabla B_{f \circ \pi(\eta)}^{o}, \nabla_{e_{j}} e_{i}\right\rangle_{x} \phi^{2}(\eta) d \mu_{o}(\eta) & \\
= & \int_{\mathcal{H} X}\left\langle\nabla B_{f \circ \pi(\eta)}^{o}, \sum_{k=1}^{n} c_{j i}^{k} e_{k}\right\rangle_{x} \phi^{2}(\eta) d \mu_{o}(\eta)=\sum_{k=1}^{n} c_{j i}^{k} Q_{k}^{\phi}(x)=0,
\end{aligned}
$$

for some constants $c_{j i}^{k}$ depending on the frame $\left\{e_{i}\right\}$. This implies the bilinear form on $T_{x} \mathbb{H}^{n}$ determined by the differential of $Q^{\phi}$ at $x$ satisfies

$$
\begin{aligned}
\left\langle v, d Q_{x}^{\phi}(v)\right\rangle & =\int_{\mathcal{H} X}\left(\operatorname{Hess} B_{f \circ \pi(\eta)}^{o}\right)_{x}(v, v) \phi^{2}(\eta) d \mu_{o}(\eta) \\
& =\int_{\partial \mathbb{H}^{n}}\left(\operatorname{Hess} B_{\theta}^{o}\right)_{x}(v, v) d\left((f \circ \pi)_{*}\left(\phi^{2} d \mu_{o}\right)\right)(\theta),
\end{aligned}
$$

for all $v \in T_{x} \mathbb{H}^{n}$. The right hand side of this equation is strictly positive by Lemma 2.1. This implies the differential of $Q^{\phi}$ at $x$ is positive definite, and thus invertible. Therefore the implicit function theorem may be applied to conclude that $P$ is $\mathcal{C}^{1}$.

Define a map

$$
\begin{aligned}
\Phi: X & \rightarrow L_{+}^{2}(\mathscr{H} X) \subset L^{2}(\mathscr{H} X) \\
x & \mapsto \sqrt{\exp \left(-h(X) b_{\eta}(x)\right)} .
\end{aligned}
$$


Lemma 5.3. $\Phi$ is Lipschitz.

Proof. Let $D$ be the diameter of the downstairs metric space $Z$ covered by $X$. It follows that for any $p \in X,\|\Phi(p)\|$ is at most $e^{D h(X)}$. Pick points $x, y \in X$. The goal is to control the quantity

$$
\int_{\mathscr{H} X}\left|e^{-\frac{1}{2} h(X) b_{\eta}(x)}-e^{-\frac{1}{2} h(X) b_{\eta}(y)}\right|^{2} d \mu_{o}(\eta) .
$$

For $\eta \in \mathscr{H} X, b_{\eta}$ is 1-Lipschitz. This implies $b_{\eta}(y) \leq d(y, x)+b_{\eta}(x)$. Using this we obtain the inequalities

$$
\begin{aligned}
e^{-\frac{1}{2} h(X) b_{\eta}(x)}-e^{-\frac{1}{2} h(X) b_{\eta}(y)} & \leq e^{-\frac{1}{2} h(X) b_{\eta}(x)}-e^{-\frac{1}{2} h(X) d(y, x)} e^{-\frac{1}{2} h(X) b_{\eta}(x)} \\
& =e^{-\frac{1}{2} h(X) b_{\eta}(x)}\left(1-e^{-\frac{1}{2} h(X) d(y, x)}\right),
\end{aligned}
$$

yielding

$$
\left|e^{-\frac{1}{2} h(X) b_{\eta}(x)}-e^{-\frac{1}{2} h(X) b_{\eta}(y)}\right| \leq\left(e^{-\frac{1}{2} h(X) b_{\eta}(x)}+e^{-\frac{1}{2} h(X) b_{\eta}(y)}\right)\left(1-e^{-\frac{1}{2} h(X) d(x, y)}\right) .
$$

Therefore,

$$
\begin{aligned}
& \int_{\mathscr{H} X}\left|e^{-\frac{1}{2} h(X) b_{\eta}(x)}-e^{-\frac{1}{2} h(X) b_{\eta}(y)}\right|^{2} d \mu_{o}(\eta) \\
& \quad \leq\left(1-e^{-\frac{1}{2} h(X) d(x, y)}\right)^{2} \int_{\mathscr{H} X}\left|e^{-\frac{1}{2} h(X) b_{\eta}(x)}+e^{-\frac{1}{2} h(X) b_{\eta}(y)}\right|^{2} d \mu_{o}(\eta) \\
& =\left(1-e^{-\frac{1}{2} h(X) d(x, y)}\right)^{2} \cdot\|\Phi(x)+\Phi(y)\|^{2} \\
& \leq\left(1-e^{-\frac{1}{2} h(X) d(x, y)}\right)^{2} \cdot[\|\Phi(x)\|+\|\Phi(y)\|]^{2} \leq\left(1-e^{-\frac{1}{2} h(X) d(x, y)}\right)^{2} \cdot 4 e^{2 D h(X)}
\end{aligned}
$$

To complete the proof, notice that for $t \geq 0,\left(1-e^{-\frac{1}{2} h(X) t}\right) \leq \frac{1}{2} h(X) t$. Applying this yields

$$
\int_{\mathscr{H} X}\left|e^{-\frac{1}{2} h(X) b(x, \eta)}-e^{-\frac{1}{2} h(X) b_{\eta}(y)}\right|^{2} d \mu_{o}(\eta) \leq\left(\frac{1}{2} h(X) d(x, y)\right)^{2} \cdot 4 e^{2 D h(X)} .
$$

So finally we've obtained

$$
\frac{\|\Phi(x)-\Phi(y)\|}{d(x, y)} \leq h(X) \cdot e^{D h(X)} .
$$

$P$ is $\mathcal{C}^{1}$, therefore $F=P \circ \Phi$ is locally Lipschitz. $\Gamma$-equivariance implies $F$ descends to a locally Lipschitz map $X / \Gamma=Z \rightarrow \mathbb{H}^{n} / \Gamma=M_{\text {hyp }}$. By Rademacher's theorem, $F$ is differentiable almost everywhere (see Section 2.5). 


\section{The Jacobian estimate}

In this section we prove parts (2) and (3) of Proposition 4.3.

(2) $|\operatorname{Jac} F(p)| \leq\left(\frac{h(X)}{n-1}\right)^{n}$ almost everywhere.

(3) If for some $p,|\operatorname{Jac} F(p)|=\left(\frac{h(X)}{n-1}\right)^{n}$, then the differential $d F_{p}$ is a homothety of ratio $\left(\frac{h(X)}{n-1}\right)^{n}$.

The proof closely follows Section 5 of $[\mathrm{F}]$, which is in turn based on [BCG2, p. 636-639]. Recall that $X$ is the universal cover of $Z, \Gamma:=\pi_{1}(Z) \cong \pi_{1}\left(M_{\text {hyp }}\right),\left\{e_{i}\right\}$ is a $\mathcal{C}^{\infty}$ frame on $T \mathbb{H}^{n}$, and $\Omega \subset X$ is a subset of full measure possessing a $\mathcal{C}^{1}$-atlas (see Sections 2.4 and 2.5).

In the previous section we have defined a $\Gamma$-equivariant locally Lipschitz map $\Phi: X \rightarrow L_{+}^{2}(\mathscr{H} X)$, a $\Gamma$-equivariant $\mathcal{C}^{1}$ barycenter map $P: L_{+}^{2}(\mathcal{H} X) \rightarrow \mathbb{H}^{n}$, and a $\mathcal{C}^{\infty}$ map $Q=\left(Q_{1}, \ldots, Q_{n}\right): \mathbb{H}^{n} \times L_{+}^{2}(\mathscr{H} X) \rightarrow \mathbb{R}^{n}$. They satisfied the equations $F=P \circ \Phi$ and $Q(P(\phi), \phi)=0$ for $\phi \in L_{+}^{2}(\mathcal{H} X)$. We thus obtain the implicit equation $Q(F, \Phi)=0$. Let $\mathcal{O} \subseteq \Omega \subseteq X$ be the set of points where $\Phi$ is differentiable. By Rademacher's theorem (see Section 2.5), $\mathcal{O} \subseteq X$ is a subset of full measure. Pick $p \in \mathcal{O}$ and $v \in T_{p} X$.

Lemma 6.1. Differentiating the function

$$
x \mapsto Q_{i}(F(x), \Phi(x))=\int_{\mathscr{H} X}\left\langle\nabla B_{f \circ \pi(\eta)}^{o}, e_{i}\right\rangle_{F(x)} e^{-h(X) b_{\eta}(x)} d \mu_{o}(\eta)=0
$$

at $p$ in the direction of $v$ yields

$$
\begin{aligned}
0= & \int_{\mathcal{H} X}\left(\operatorname{Hess} B_{f \circ \pi(\eta)}^{o}\right)_{F(p)}\left(d F(v), e_{i}\right) e^{-h(X) b_{\eta}(p)} d \mu_{o}(\eta) \\
& +\int_{\mathcal{H} X}\left\langle\nabla B_{f \circ \pi(\eta)}^{o}, \nabla_{d F(v)} e_{i}\right\rangle_{F(p)} e^{-h(X) b_{\eta}(p)} d \mu_{o}(\eta) \\
& +\int_{\mathscr{H} X}\left\langle\nabla B_{f \circ \pi(\eta)}^{o}, e_{i}\right\rangle_{F(p)}\left[-h(X)\left\langle\nabla b_{\eta}, v\right\rangle_{p} e^{-h(X) b_{\eta}(p)}\right] d \mu_{o}(\eta) .
\end{aligned}
$$

Proof. This is an application of the Lebesgue dominated convergence theorem. $\mathscr{H} X$ is compact. So to apply the theorem it is sufficient to find a $c>0$ such that for all $\eta \in \mathscr{H} X$, the function

$$
x \mapsto\left\langle\nabla B_{f \circ \pi(\eta)}^{o}, e_{i}\right\rangle_{F(x)} e^{-h(X) b_{\eta}(x)}
$$

is locally $c$-Lipschitz near $p$. To show the existence of such a constant, use that $b_{\eta}$ is 1-Lipschitz, $\mathscr{H} X$ is compact, and $\left\langle\nabla B_{\theta}^{o}, e_{i}\right\rangle_{q}: \mathbb{H}^{n} \times \partial \mathbb{H}^{n} \rightarrow \mathbb{R}$ is $\mathcal{C}^{\infty}$. 
The second term in equation $(\star)$ satisfies

$$
\begin{aligned}
\int_{\mathcal{H} X}\left\langle\nabla B_{f \circ \pi(\eta)}^{o}, \nabla_{d F(v)} e_{i}\right\rangle_{F(p)} e^{-h(X) b_{\eta}(p)} d \mu_{o}(\eta) \\
=\int_{\mathcal{H} X}\left\langle\nabla B_{f \circ \pi(\eta)}^{o}, \sum_{j=1}^{n} c_{i j} e_{j}\right\rangle_{F(p)} e^{-h(X) b_{\eta}(p)} d \mu_{o}(\eta) \\
=\sum_{j=1}^{n} c_{i j} Q_{j}(F(p), \Phi(p))=0,
\end{aligned}
$$

for some constants $c_{i j}$ depending on the frame $\left\{e_{i}\right\}$. Therefore, for $v \in T_{p} X, u \in$ $T_{F(p)} \mathbb{H}^{n}$ we have

$$
\begin{aligned}
& \int_{\mathscr{H} X}\left(\operatorname{Hess} B_{f \circ \pi(\eta)}^{o}\right)_{F(p)}(d F(v), u) e^{-h(X) b_{\eta}(p)} d \mu_{o}(\eta) \\
& \quad=h(X) \int_{\mathscr{H} X}\left\langle\nabla B_{f \circ \pi(\eta)}^{o}, u\right\rangle_{F(p)}\left\langle\nabla b_{\eta}, v\right\rangle_{p} e^{-h(X) b_{\eta}(p)} d \mu_{o}(\eta) .
\end{aligned}
$$

Let $\left\|\mu_{p}\right\|$ denote the total mass $\mu_{p}(\mathcal{H} X)$ of the measure $\mu_{p}$. Define a bilinear form $K$ and a quadratic form $H$ on $T_{F(p)} \mathbb{H}^{n}$ by

$$
\begin{gathered}
\langle K(w), u\rangle_{F(p)}:=\frac{1}{\left\|\mu_{p}\right\|} \int_{\mathscr{H} X}\left(\operatorname{Hess} B_{f \circ \pi(\eta)}^{o}\right)_{F(p)}(w, u) e^{-h(X) b_{\eta}(p)} d \mu_{o}(\eta) \\
\langle H(u), u\rangle_{F(p)}:=\frac{1}{\left\|\mu_{p}\right\|} \int_{\mathscr{H} X}\left\langle\nabla B_{f \circ \pi(\eta)}^{o}, u\right\rangle_{F(p)}^{2} e^{-h(X) b_{\eta}(p)} d \mu_{o}(\eta) .
\end{gathered}
$$

Note that the symmetric endomorphism $K$ is positive definite by Theorem 2.1. It is therefore invertible. This is used in the proof of Lemma 6.2.

Use equation $(\star \star)$, the Cauchy-Schwarz inequality, and the definition of $K$ to obtain

$$
\begin{aligned}
& \mid\langle K \circ dd F(v), u\rangle_{F(p)} \mid \\
& \quad=\left|\frac{h(X)}{\left\|\mu_{p}\right\|} \int_{\mathscr{H} X}\left\langle\nabla B_{f \circ \pi(\eta)}^{o}, u\right\rangle_{F(p)} \cdot\left\langle\nabla b_{\eta}, v\right\rangle_{p} \cdot e^{-h(X) b_{\eta}(p)} d \mu_{o}(\eta)\right| \\
& \quad \leq \frac{h(X)}{\left\|\mu_{p}\right\|}\left[\int_{\mathscr{H} X}\left\langle\nabla B_{f \circ \pi(\eta)}^{o}, u\right\rangle_{F(p)}^{2} d \mu_{p}(\eta)\right]^{1 / 2} \cdot\left[\int_{\mathscr{H} X}\left\langle\nabla b_{\eta}, v\right\rangle_{p}^{2} d \mu_{p}(\eta)\right]^{1 / 2} \\
& \quad=\frac{h(X)}{\left\|\mu_{p}\right\|^{1 / 2}}\left[\langle H(u), u\rangle_{F(p)}\right]^{1 / 2} \cdot\left[\int_{\mathscr{H} X}\left\langle\nabla b_{\eta}, v\right\rangle_{p}^{2} d \mu_{p}(\eta)\right]^{1 / 2},
\end{aligned}
$$

for all $u \in T_{F(p)} \mathbb{H}^{n}$ and $v \in T_{p} \Omega$. 
Lemma 6.2 ([BCG2, p. 637]). For all $p \in \mathcal{O}$,

$$
|\operatorname{Jac} F(p)| \leq \frac{h(X)^{n}(\operatorname{det} H)^{1 / 2}}{n^{n / 2} \operatorname{det} K} .
$$

Proof. The proof is the proof of Lemma 5.4 of [BCG2, p. 637] with two modifications. First replace the Busemann function $B_{\alpha}$ with the horofunction $b_{\eta}$, and notice that

$$
\sum_{i=1}^{n}\left\langle\nabla b_{\eta}, v_{i}\right\rangle_{p}^{2}=\left\|\nabla b_{\eta}\right\|_{p}^{2} \leq 1 .
$$

Second, the total mass of the Patterson-Sullivan measure $\left\|\mu_{p}\right\|$ must be carried through the estimate, but it cancels itself out in the final step.

A key property of hyperbolic space is that Busemann functions on $\mathbb{H}^{n}$ satisfy the equation

$$
\left(\operatorname{Hess} B_{\theta}^{o}\right)(u, v)=\langle u, v\rangle-\left\langle\nabla B_{\theta}^{o}, u\right\rangle \cdot\left\langle\nabla B_{\theta}^{o}, v\right\rangle
$$

for all $\theta \in \partial \mathbb{H}^{n}$ (see [BCG1, p. 750-751]). Integrating this equation over $\mathscr{H} X$ yields

$$
K=\mathrm{Id}-H .
$$

Lemma 6.3. The symmetric endomorphism $H$ is positive definite.

Proof. Suppose there exists $x \in X$ and $u \in T_{x} X$ such that $\langle H(u), u\rangle_{x}=0$. From the definition of $H$, this implies the support of the measure $(f \circ \pi)_{*} \mu_{0}$ on $\partial \mathbb{H}^{n}$ is contained in a codimension one conformally round sphere in $S \subset \mathbb{H}^{n}$. By the equivariance of the Patterson-Sullivan measures, the support of $(f \circ \pi)_{*} \mu_{\gamma . o}$ is contained in $\gamma(S)$ for $\gamma \in \Gamma$. But $\mu_{\gamma . o} \ll \mu_{o}$ implies the support of $(f \circ \pi)_{*} \mu_{\gamma . o}$ is contained in $S$. Therefore $\Gamma$ preserves $S \subset \partial \mathbb{H}^{n}$. This contradicts the fact that the limit set of $\Gamma$ is the entire sphere at infinity.

A short computation shows that $\operatorname{tr}(H)=1$. Therefore we can apply the brain in a jar lemma (Lemma 2.2) to $H$. This yields

$$
\frac{\operatorname{det}(H)}{[\operatorname{det}(\operatorname{Id}-H)]^{2}} \leq\left[\frac{n}{(n-1)^{2}}\right]^{n},
$$

with equality if and only if $H=\frac{1}{n} \mathrm{Id}$. Combining this with Lemma 6.2 proves the desired inequality, namely

$$
|\operatorname{Jac} F(p)| \leq\left(\frac{h(X)}{n-1}\right)^{n} .
$$

This completes the proof of part (2) of Proposition 4.3. 
The proof of part (3) of Proposition 4.3 is the "equality case" argument on page 639 of [BCG2] with two modifications (as in Lemma 6.2). First replace the Busemann function $B_{\alpha}$ with the horofunction $b_{\eta}$ and use inequality ( $\ddagger$ ). Second, the total mass of the Patterson-Sullivan $\left\|\mu_{p}\right\|$ must be carried through the estimate, and again it cancels itself out. This completes the proof of Proposition 4.3.

\section{The equality case}

Theorem 7.1. Let $M_{\text {hyp }}$ be a closed hyperbolic $n$-manifold for $n \geq 3$. Let $Z$ be a compact $n$-dimensional convex Riemannian amalgam (see Section 2.8). Let $f: Z \rightarrow M_{\text {hyp }}$ be a homotopy equivalence. If

$$
h(X)^{n} \operatorname{Vol}(Z)=(n-1)^{n} \operatorname{Vol}\left(M_{\mathrm{hyp}}\right)
$$

then the natural map $F: Z \rightarrow M_{\mathrm{hyp}}$ is a homothetic homeomorphism.

Remark 7.2. Recall that if $Z$ is either

- the metric doubling of a convex hyperbolic manifold with boundary (see Section 2.7), or

- a cone-manifold (see Section 2.6),

then $Z$ is a convex Riemannian amalgam.

Let $X$ be the universal cover of $Z$. Lift the convex Riemannian amalgam structure on $Z$ to a convex Riemannian amalgam structure on $X$ with decomposition $\left\{C_{j}\right\}$ into convex Riemannian manifolds with boundary. Define the incomplete disconnected Riemannian manifold $\Omega:=\bigcup_{j} \operatorname{int}\left(C_{j}\right) \subseteq X$.

The equation

$$
h(X)^{n} \operatorname{Vol}(Z)=(n-1)^{n} \operatorname{Vol}\left(M_{\mathrm{hyp}}\right)
$$

implies the string of inequalities from Lemma 4.4 is in fact a string of equalities. Therefore $|\operatorname{Jac} F(p)|=\left(\frac{h(X)}{n-1}\right)^{n}$ almost everywhere. By Proposition 4.3, $d F$ is a homothety almost everywhere. The goal is to prove $F: Z \rightarrow M_{\text {hyp }}$ is a homothetic homeomorphism. Without a loss of generality, scale the metric of $Z$ so that $d F$ is an isometry (not necessarily orientation preserving) almost everywhere. This forces $\operatorname{Vol}(Z)=\operatorname{Vol}\left(M_{\text {hyp }}\right)$. It now suffices to show that $F: Z \rightarrow M_{\text {hyp }}$ is an isometric homeomorphism. This will be done by working on the universal covers and showing $F: X \rightarrow \mathbb{H}^{n}$ is an equivariant isometric homeomorphism.

Lemma 7.3. $F: X \rightarrow \mathbb{H}^{n}$ is a contraction mapping, i.e. for any pair $x, y \in X$, $d_{\mathbb{H}^{n}}(F(x), F(y)) \leq d_{X}(x, y)$. 
Proof. Pick a length minimizing geodesic segment $\alpha$ connecting $x$ to $y$. Define $\mathcal{O} \subseteq \Omega \subseteq X$ to be the set of full measure where $d F$ is an isometry. There exists a small perturbation $\alpha_{\varepsilon}$ of $\alpha$ such that $\alpha_{\varepsilon} \cap \mathcal{O} \subseteq \alpha_{\varepsilon}$ is a subset of full measure, and length $\left(\alpha_{\varepsilon}\right) \leq$ length $(\alpha)+\varepsilon$. $F$ is locally Lipschitz, so in all Hausdorff dimensions $F$ maps measure zero sets to measure zero sets. This implies $F$ preserves length on $\alpha_{\varepsilon}$. Therefore

$d_{\mathbb{H}^{n}}(F(x), F(y)) \leq$ length $\left(F\left(\alpha_{\varepsilon}\right)\right)=$ length $\left(\alpha_{\varepsilon}\right) \leq$ length $(\alpha)+\varepsilon=d_{X}(x, y)+\varepsilon$.

Since $\varepsilon$ was arbitrary, the result follows.

We claim that $F$ is volume preserving. $\mid$ Jac $F \mid=1$ a.e. and $F$ is locally Lipschitz, so $F$ is volume non-increasing. If it strictly decreased the volume of some measurable set, then we would have $\operatorname{Vol}(Z)>\operatorname{Vol}\left(M_{\text {hyp }}\right)$. This would be a contradiction. Therefore $F$ is volume preserving.

There exist constants $C_{1}, \varepsilon_{\text {hyp }}>0$ such that if $y \in \mathbb{H}^{n}$ and $\varepsilon<\varepsilon_{\text {hyp }}$ then

$$
v_{n} \varepsilon^{n} \leq \operatorname{Vol}\left(B_{\mathbb{H}^{n}}(y, \varepsilon)\right) \leq v_{n} \varepsilon^{n}\left(1+C_{1} \varepsilon^{2}\right),
$$

where $v_{n}$ is the volume of a unit ball in $\mathbb{R}^{n}$. Similarly, $\Omega \subseteq X$ is a Riemannian manifold with sectional curvature bounded from above. (An upper curvature bound follows from the definition of a convex Riemannian amalgam.) For compact $K \subset \Omega$ (possibly a point), define

$$
\operatorname{inj}_{\Omega}(K):=\inf _{z \in K}(\text { injectivity radius of } \Omega \text { at } z \text { ). }
$$

The upper curvature bound implies there exist constants $C_{2}, \varepsilon_{\Omega}>0$ such that if $\varepsilon<\varepsilon_{\Omega}, \varepsilon<\operatorname{inj}_{\Omega}(z)$, and $B_{X}(z, \varepsilon) \subset \Omega$ then

$$
v_{n} \varepsilon^{n}\left(1-C_{2} \varepsilon^{2}\right) \leq \operatorname{Vol}\left(B_{\Omega}(z, \varepsilon)\right) .
$$

We now consider the restriction of $F$ to the "smooth" set $\Omega$. Define $F_{\Omega}:=$ $\left.F\right|_{\Omega}: \Omega \rightarrow \mathbb{H}^{n}$.

Lemma 7.4. $F_{\Omega}$ is injective.

Proof. (This proof is an adaptation of [BCG1, Lemma C.4].) Suppose there exist distinct $z_{1}, z_{2} \in \Omega$ such that $F_{\Omega}\left(z_{1}\right)=F_{\Omega}\left(z_{2}\right)=y$. Pick

$$
\varepsilon_{0}<\min \left\{\varepsilon_{\Omega}, \operatorname{inj}_{\Omega}\left(z_{1}\right), \operatorname{inj}_{\Omega}\left(z_{2}\right), \varepsilon_{\mathrm{hyp}}\right\}
$$

sufficiently small such that

$$
B_{X}\left(z_{1}, \varepsilon_{0}\right) \cap B_{X}\left(z_{2}, \varepsilon_{0}\right)=\emptyset \quad \text { and } \quad B_{X}\left(z_{1}, \varepsilon_{0}\right) \cup B_{X}\left(z_{2}, \varepsilon_{0}\right) \subset \Omega .
$$


In particular, inequality 4 (resp. inequality 5) is valid at $y$ (resp. $z_{1}$ and $z_{2}$ ) for $\varepsilon<\varepsilon_{0}$. Since $F$ is contracting, $F_{\Omega}\left(B_{\Omega}\left(z_{i}, \varepsilon\right)\right) \subseteq B_{\mathbb{H}^{n}}(y, \varepsilon)$ for all $\varepsilon<\varepsilon_{0}$. Therefore

$$
B_{\Omega}\left(z_{1}, \varepsilon\right) \cup B_{\Omega}\left(z_{2}, \varepsilon\right) \subseteq F_{\Omega}^{-1}\left(B_{\mathbb{H}^{n}}(y, \varepsilon)\right) .
$$

Since $F$ is volume preserving (and $X \backslash \Omega$ is measure zero),

$$
\begin{aligned}
\operatorname{Vol}\left(B_{\mathbb{H}^{n}}(y, \varepsilon)\right)=\operatorname{Vol}\left(F_{\Omega}^{-1}\left(B_{\mathbb{H}^{n}}(y, \varepsilon)\right)\right) & \geq \operatorname{Vol}\left(B_{\Omega}\left(z_{1}, \varepsilon\right) \cup B_{\Omega}\left(z_{2}, \varepsilon\right)\right) \\
& =\operatorname{Vol}\left(B_{\Omega}\left(z_{1}, \varepsilon\right)\right)+\operatorname{Vol}\left(B_{\Omega}\left(z_{2}, \varepsilon\right)\right) .
\end{aligned}
$$

Now apply inequalities 4 and 5 to obtain

$$
v_{n} \varepsilon^{n}\left(1+C_{1} \varepsilon^{2}\right) \geq 2 v_{n} \varepsilon^{n}\left(1-C_{2} \varepsilon^{2}\right) .
$$

This implies $\varepsilon^{2}\left(C_{1}+2 C_{2}\right) \geq 1$ for all $\varepsilon<\varepsilon_{0}$, which is a contradiction.

Since $\Omega$ is locally compact, Lemma 7.4 shows that $F_{\Omega}: \Omega \rightarrow F_{\Omega}(\Omega)$ is a homeomorphism.

Lemma 7.5. $F_{\Omega}^{-1}$ is locally Lipschitz.

Proof. (This proof is an adaptation of [BCG1, Lemma C.7].) Pick $z \in \Omega$. Let $y:=F_{\Omega}(z)$. Pick $\varepsilon_{0}<\min \left\{\varepsilon_{\text {hyp }}, \varepsilon_{\Omega}\right\}$ sufficiently small such that $B_{X}\left(z, 3 \varepsilon_{0}\right) \subset \Omega$, $\varepsilon_{0}<\operatorname{inj}_{\Omega}\left(\overline{B_{X}\left(z, 2 \varepsilon_{0}\right)}\right)$, and

$$
2 \varepsilon_{0}^{2}\left(C_{1}+C_{2}\right)<\frac{1}{2^{n}}
$$

Define $V:=B_{\Omega}\left(z, \varepsilon_{0}\right)$. Since $F$ is a contraction mapping, $F(V) \subseteq B_{\mathbb{H}^{n}}\left(y, \varepsilon_{0}\right)$. We will show that if $z_{1}, z_{2} \in V$ are distinct, then

$$
d_{X}\left(z_{1}, z_{2}\right)<2 d_{\mathbb{H}^{n}}\left(F\left(z_{1}\right), F\left(z_{2}\right)\right) .
$$

This will imply $\left.F\right|_{V}{ }^{-1}$ is 2-Lipschitz on the open set $F(V)$.

Suppose the above inequality is false, i.e. there exist distinct $z_{1}, z_{2} \in V$ such that $2 d_{\mathbb{H}^{n}}\left(F\left(z_{1}\right), F\left(z_{2}\right)\right) \leq d_{X}\left(z_{1}, z_{2}\right)$. Set $\varepsilon:=d_{\mathbb{H}^{n}}\left(F\left(z_{1}\right), F\left(z_{2}\right)\right) \leq \varepsilon_{0}$. Since $\varepsilon<\varepsilon_{0}$, inequality 4 remains valid at $F\left(z_{1}\right)$ and $F\left(z_{2}\right)$ for $\varepsilon$. $B_{\mathbb{H}^{n}}\left(F\left(z_{1}\right), \varepsilon\right)$ and $B_{\mathbb{H}^{n}}\left(F\left(z_{2}\right), \varepsilon\right)$ intersect, and their intersection contains a ball of radius $\varepsilon / 2$ centered in the middle of the minimizing geodesic joining $F\left(z_{1}\right)$ to $F\left(z_{2}\right)$ (see Figure 5). Therefore

$$
\operatorname{Vol}\left(B_{\mathbb{H}^{n}}\left(F\left(z_{1}\right), \varepsilon\right) \cup B_{\mathbb{H}^{n}}\left(F\left(z_{2}\right), \varepsilon\right)\right) \leq v_{n}\left[2 \varepsilon^{n}\left(1+C_{1} \varepsilon^{2}\right)-\frac{1}{2^{n}} \varepsilon^{n}\right] .
$$

Moreover, $d_{X}\left(z_{1}, z_{2}\right) \geq 2 \varepsilon$ implies $B_{X}\left(z_{1}, \varepsilon\right) \cap B_{X}\left(z_{2}, \varepsilon\right)=\emptyset$. Since $B_{X}\left(z_{1}, \varepsilon\right) \cup$ $B_{X}\left(z_{2}, \varepsilon\right) \subset B\left(z, 2 \varepsilon_{0}\right) \subset \Omega$, we may apply inequality 5 to conclude

$$
\begin{aligned}
\operatorname{Vol}\left(F\left(B_{X}\left(z_{1}, \varepsilon\right) \cup B_{X}\left(z_{2}, \varepsilon\right)\right)\right) & =\operatorname{Vol}\left(B_{X}\left(z_{1}, \varepsilon\right) \cup B_{X}\left(z_{2}, \varepsilon\right)\right) \\
& \geq v_{n}\left[2 \varepsilon^{n}\left(1-C_{2} \varepsilon^{2}\right)\right] .
\end{aligned}
$$




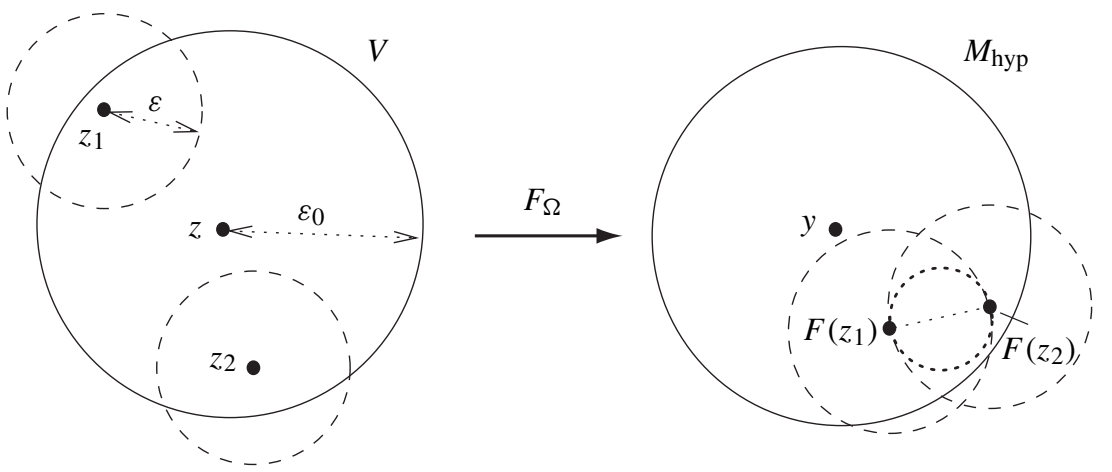

Figure 5

$F$ is a contraction mapping. Therefore

$$
F\left(B_{X}\left(z_{1}, \varepsilon\right)\right) \subseteq B_{\mathbb{H}^{n}}\left(F\left(z_{1}\right), \varepsilon\right) \quad \text { and } \quad F\left(B_{X}\left(z_{2}, \varepsilon\right)\right) \subseteq B_{\mathbb{H}^{n}}\left(F\left(z_{2}\right), \varepsilon\right)
$$

Putting these inequalities together yields

$$
v_{n}\left[2 \varepsilon^{n}\left(1+C_{1} \varepsilon^{2}\right)-\frac{1}{2^{n}} \varepsilon^{n}\right] \geq v_{n} \varepsilon^{n}\left[2\left(1-C_{2} \varepsilon^{2}\right)\right],
$$

implying

$$
2 \varepsilon^{2}\left(C_{1}+C_{2}\right) \geq \frac{1}{2^{n}}
$$

This contradicts our choice of $\varepsilon_{0}$.

Since $\left.F\right|_{\Omega} ^{-1}$ is locally Lipschitz, it is differentiable almost everywhere. Therefore, for almost every $z \in \Omega$,

$$
d\left(\operatorname{Id}_{\Omega}\right)_{z}=d\left(\left.F\right|_{\Omega} ^{-1} \circ F_{\Omega}\right)_{z}=d\left(\left.F\right|_{\Omega} ^{-1}\right) \circ d F_{\Omega} .
$$

This implies $d\left(\left.F\right|_{\Omega^{-1}}\right)$ is an isometry of tangent spaces almost everywhere. By an argument analogous to Lemma 7.3, and by working on small balls in $M_{\text {hyp }}$, one can see that $\left.F\right|_{\Omega} ^{-1}$ is locally a contraction mapping $F(\Omega) \rightarrow \Omega$. Both $F_{\Omega}$ and $\left.F\right|_{\Omega}{ }^{-1}$ are local contraction maps. Thus they are both local isometries.

Lemma 7.6. For all $j,\left.F\right|_{C_{j}}$ is an isometry onto its image, and $F\left(\operatorname{int}\left(C_{j}\right)\right)$ is convex.

Proof. By continuity, it suffices to show $\left.F\right|_{\operatorname{int}\left(C_{j}\right)}$ is an isometry onto its image. Pick $x, y \in \operatorname{int}\left(C_{j}\right)$. Since int $\left(C_{j}\right)$ is convex, there exists a geodesic segment $x y \subset \operatorname{int}\left(C_{j}\right)$ joining $x$ to $y . \quad F_{\Omega}$ is a local isometry. Thus length $(F(x y))=\operatorname{length}(x y)$, and 
$F(x y) \subset \mathbb{H}^{n}$ is locally a hyperbolic geodesic joining $F(x)$ to $F(y)$. In $\mathbb{H}^{n}$, a local geodesic is a global geodesic. Therefore

$$
d_{\mathbb{H}^{n}}(F(x), F(y))=\text { length }(F(x y))=\text { length }(x y)=d_{X}(x, y) .
$$

Lemma 7.7. For each $z \in X$, there exists $\delta_{z}>0$ such that $d_{X}(z, x)<\delta_{z}$ implies $d_{\mathbb{H}^{n}}(F(z), F(x))=d_{X}(z, x)$.

(Notice that this lemma proves neither that $F$ is an isometry nor that it is locally injective. To see why, consider the branched double covering of an Euclidean disk by an Euclidean cone with cone angle $4 \pi$.)

Proof. Pick $z \in X$. If $z \in \Omega$ then we are done. So assume $z \in X \backslash \Omega$. Up to rearranging the indices we may assume

$$
z \in\left(\partial C_{1} \cup \partial C_{2} \cup \cdots \cup \partial C_{l}\right) \backslash\left(\partial C_{l+1} \cup \cdots \cup \partial C_{N}\right) .
$$

By this, there exists $\delta_{z}>0$ such that

$$
B_{X}\left(z, \delta_{z}\right) \cap \Omega \subseteq \bigcup_{j=1}^{l} C_{j} .
$$

For $x \in B_{X}\left(z, \delta_{z}\right)$, there exists an integer $j_{x}$ and a geodesic segment $x z \subset C_{j_{x}} .\left.F\right|_{C_{j_{x}}}$ is an isometry, so $d_{X}(z, x)=d_{C_{j_{x}}}(z, x)=d_{\mathbb{H}^{n}}(F(z), F(x))$. This proves the lemma.

As a corollary of this lemma, we see that $F^{-1}(y)$ is a discrete set for all $y \in \mathbb{H}^{n}$.

Lemma 7.8. $F: X \rightarrow \mathbb{H}^{n}$ is injective.

Proof. For $y \in \mathbb{H}^{n}, F^{-1}(y)$ is the discrete set $\left\{z_{i}\right\}$. We will show $F^{-1}(y)$ must be a single point. For all $i$, pick $\delta_{i}<1$ so that $d_{X}\left(z_{i}, x\right)<\delta_{i}$ implies $d_{\mathbb{H}^{n}}\left(F\left(z_{i}\right), F(x)\right)=$ $d_{X}\left(z_{i}, x\right)$. Assume the metric balls $B_{X}\left(z_{i}, \delta_{i}\right)$ are disjoint.

If for some $i,\left.F\right|_{B_{X}\left(z_{i}, \delta_{i}\right)}: B_{X}\left(z_{i}, \delta_{i}\right) \rightarrow B_{\mathbb{H}^{n}}\left(F\left(z_{i}\right), \delta_{i}\right)$ is not surjective, then $F$ can be properly homotoped to a map taking $B_{X}\left(z_{i}, \delta_{i}\right) \rightarrow \partial B_{\mathbb{H}^{n}}\left(F\left(z_{i}\right), \delta_{i}\right)$. Moreover, this can be done without altering $F$ outside of $B_{X}\left(z_{i}, \delta_{i}\right)$.

If for every $i,\left.F\right|_{B_{X}\left(z_{i}, \delta_{i}\right)}: B_{X}\left(z_{i}, \delta_{i}\right) \rightarrow B_{\mathbb{H}^{n}}\left(F\left(z_{i}\right), \delta_{i}\right)$ is not surjective, then $F$ is properly homotopic to a map which does not have $y$ in its image. $F$ is a proper surjective homotopy equivalence. Thus every map properly homotopic to $F$ is surjective. Therefore for some $i,\left.F\right|_{B_{X}\left(z_{i}, \delta_{i}\right)}$ is surjective. We may assume $i=1$.

$F_{\Omega}$ is injective and open, $\Omega$ is open and dense, and $F\left(B_{X}\left(z_{1}, \delta_{1}\right)\right)=B_{\mathbb{H}^{n}}\left(y, \delta_{1}\right)$. Thus

$$
F\left(\Omega \backslash B_{X}\left(z_{1}, \delta_{1}\right)\right) \cap B_{\mathbb{H}^{n}}\left(y, \delta_{1}\right)=\emptyset .
$$


By extending to the closure of $\Omega$, this shows that for all $i>1$,

$$
F\left(z_{i}\right)=y \notin B_{\mathbb{H}^{n}}\left(y, \delta_{1}\right) .
$$

Therefore $F^{-1}(y)$ must be the single point $z_{1}$.

Recall that $F: X \rightarrow \mathbb{H}^{n}$ is surjective. Therefore $F: X \rightarrow \mathbb{H}^{n}$ is a continuous bijection. Since $X$ is locally compact, $F$ is a homeomorphism. To prove that $F$ is an isometry, it is sufficient to show $F^{-1}$ is a contraction mapping.

Lemma 7.9. $F^{-1}$ is a contraction mapping, i.e. it is 1-Lipschitz.

Proof. By the above lemmas, $F$ imposes a convex Riemannian amalgam structure on $\mathbb{H}^{n}$. The collection of convex Riemannian manifolds with boundary is $\left\{F\left(C_{j}\right)\right\}$.

Pick $x, y \in \mathbb{H}^{n}, \varepsilon>0$. Since each $F\left(C_{j}\right)$ is convex, there exists a path $\gamma \subset \mathbb{H}^{n}$ joining $x$ to $y$ such that:

- length $(\gamma) \leq d(x, y)+\varepsilon$,

- $\gamma \cap F\left(\partial C_{j}\right)$ is at most two points for any $F\left(C_{j}\right)$ of the decomposition. As the collection $\left\{F\left(C_{j}\right)\right\}$ is locally finite, the set $F^{-1}(\gamma) \backslash \Omega$ is finite. $\left.F\right|_{C_{j}}$ is an isometry for all $j$. Therefore $F^{-1}(\gamma)$ is a path of the same length as $\gamma$. This implies $F^{-1}$ is 1-Lipschitz.

This completes the proof of Theorem 7.1.

\section{Applications}

8.1. Kleinian groups. The Kleinian group theory notation used here is defined in Sections 2.9-2.12.

Let $N$ be a compact acylindrical 3-manifold (see Section 2.10). Recall that by Corollary 2.5, there exists a convex cocompact hyperbolic 3-manifold $M_{g}$ such that $C_{M_{g}}$ is homeomorphic to $N$ and the boundary of the convex core $\partial C_{M_{g}} \subset M_{g}$ is totally geodesic.

As was discussed in Section 1, the following theorem solves a conjecture in Kleinian groups.

Theorem 8.1. Let $N$ be a compact acylindrical 3-manifold. Let $\left(M_{g}, m_{g}\right)$ be a convex cocompact hyperbolic 3-manifold such that $C_{M_{g}}$ is homeomorphic to $N$ and the boundary of the convex core $\partial C_{M_{g}} \subset M_{g}$ is totally geodesic. For all $(M, m) \in \mathrm{H}(N)$,

$$
\operatorname{Vol}\left(C_{M}\right) \geq \operatorname{Vol}\left(C_{M_{g}}\right),
$$

with equality if and only if $M$ and $M_{g}$ are isometric. 
Fix an $(M, m) \in \mathrm{H}(N)$. We may assume without a loss of generality that $M$ is geometrically finite. Since $\partial C_{M_{g}}$ is totally geodesic, metrically doubling $C_{M_{g}}$ across its boundary produces a compact hyperbolic manifold $D C_{M_{g}}$.

Lemma 8.2. Metrically doubling the convex core $C_{M}$ across its boundary yields an Alexandrov space with curvature bounded below by -1 .

Proof. In [S, Appendix A] it was proven that taking an $\varepsilon$-neighborhood of $C_{M}$ in $M$, and metrically doubling that across its boundary to obtain $D \mathcal{N}_{\varepsilon} C_{M}$, yields an Alexandrov space with curvature bounded below by $-1 . D C_{M}$ is the Gromov-Hausdorff limit of these spaces as $\varepsilon \rightarrow 0$. Being an Alexandrov space with curvature bounded below by -1 is a closed property in the Gromov-Hausdorff topology [BBI, Proposition 10.7.1, p. 376]. This proves the lemma.

Remark 8.3. Lemma 8.2 also follows from a more general theorem of Perelman (see Theorem 8.8).

In particular, by Lemma 8.2 and Theorem 2.7 , the volume growth entropy of $\widetilde{D C_{M}}$ is not greater than $2=h\left(\mathbb{H}^{3}\right)$. This will be used later. Let $\sigma$ denote the boundary preserving isometric involution of $D C_{M}$ and $D C_{M_{g}}$.

Case 1. Assume $M$ is convex cocompact.

Proof of Case 1. Both $M$ nor $M_{g}$ are convex cocompact. By Theorem 2.4, $m_{g} \circ m^{-1}$ is homotopic to a homeomorphism $C_{M} \rightarrow C_{M_{g}}$. $\left(m^{-1}\right.$ denotes a relative homotopy inverse of $m$.) This homeomorphism can be "doubled" to produce a homeomorphism between the doubled manifolds $f: D C_{M} \rightarrow D C_{M_{g}}$. Theorem 4.1 may now be applied to $f: D C_{M} \rightarrow D C_{M_{g}}$. This proves that

$$
h\left(\widetilde{D C_{M}}\right)^{3} \operatorname{Vol}\left(D C_{M}\right) \geq 2^{3} \operatorname{Vol}\left(D C_{M_{g}}\right),
$$

with equality if and only if $D C_{M}$ and $D C_{M_{g}}$ are isometric. Since $h\left(\widetilde{D C_{M}}\right) \leq 2$ we have

$$
\operatorname{Vol}\left(D C_{M}\right) \geq \operatorname{Vol}\left(D C_{M_{g}}\right),
$$

with equality if and only if $D C_{M}$ and $D C_{M_{g}}$ are isometric. Dividing both sides by 2 yields the desired inequality.

Let us now assume $\operatorname{Vol}\left(D C_{M}\right)=\operatorname{Vol}\left(D C_{M_{g}}\right)$. The goal is to show $M$ and $M_{g}$ are isometric. To do this it is sufficient to show $C_{M}$ and $C_{M_{g}}$ are isometric. The map $f: D C_{M} \rightarrow D C_{M_{g}}$ is by construction $\sigma$-equivariant. Let $F: D C_{M} \rightarrow D C_{M_{g}}$ be the natural map induced by $f$. The $\sigma$-equivariance of $f$ implies $F$ is also $\sigma$-equivariant (see Remark 4.2). Therefore $F: C_{M} \rightarrow C_{M_{g}}$ is an isometry. This completes the proof of Case 1 . 
Case 2. $(M, m)$ is not convex cocompact.

Proof of Case 2. $(M, m) \in \mathrm{H}(N)$, so by definition $m: N \rightarrow M^{o}$ is a homotopy equivalence (see Section 2.12). Moreover, by Theorem 2.4 there exists a homeomorphism $g: N \rightarrow C_{M} \cap M^{o}$. Let $P:=g^{-1}\left(C_{M} \cap \partial M^{o}\right) \subset \partial N$. Consider $(M, m)$ as an element of $\mathrm{H}(N, P)$ with no additional parabolics. $(N, P)$ is pared acylindrical. Therefore there exists a geometrically finite $M_{P} \in \mathrm{H}(N, P)$ with no additional parabolics such that $C_{M_{P}}$ has totally geodesic boundary.

Again by Theorem 2.4, there is a homeomorphism $C_{M_{P}} \rightarrow N \backslash P$, inducing a doubled homeomorphism $D C_{M_{P}} \rightarrow D(N \backslash P)$ between open manifolds. Let $p \subset P \subset \partial N$ be a finite collection of disjoint simple closed curves such that $p$ is a strong deformation retract of $P$, i.e. each component of $p$ is a core curve of a component of $P$. Then there is a homeomorphism $D(N \backslash P) \rightarrow(D N) \backslash p$. Moreover, the manifolds $D C_{M_{P}} \cong D(N \backslash P)$ are topologically obtained by removing the curves $p \subset \partial N \subset D N$. Conversely, replacing the removed curves of $D N \backslash p$ corresponds to performing a (topological) Dehn surgery on $D N \backslash p$. Therefore the homeomorphism type of $D N \cong D C_{M_{g}}$ can be obtained by performing a topological Dehn surgery on $D C_{M_{P}} . D C_{M_{P}}$ is a finite volume hyperbolic manifold. Therefore Mostow rigidity implies that $D C_{M_{g}}$ may in fact be obtained by performing a hyperbolic Dehn surgery on $D C_{M_{P}}$ [Th1].

Hyperbolic Dehn surgery strictly decreases volume [Th1, Theorem 6.5.6], [Bes]. Therefore $\operatorname{Vol}\left(D C_{M_{P}}\right)>\operatorname{Vol}\left(D C_{M_{g}}\right)$. Moreover, by [NZ] there exists a closed hyperbolic 3-manifold $L$ obtained from hyperbolic Dehn surgery on $D C_{M_{P}}$ such that

$$
\operatorname{Vol}\left(D C_{M_{P}}\right)>\operatorname{Vol}(L)>\operatorname{Vol}\left(D C_{M_{g}}\right) .
$$

So to complete the proof it suffices to show that

$$
\operatorname{Vol}\left(D C_{M}\right) \geq \operatorname{Vol}(L) .
$$

This will be accomplished by geometrically filling in the cusps and reducing to the case of closed manifolds.

The geometric (though not hyperbolic) Dehn surgery arguments below are based on techniques from [Bes], [L]. (See also [BCS] for another application of these methods.) The exposition here will roughly follow [Bes].

Outside a compact set, the metric on $D C_{M}$ is a collection of smooth rank two hyperbolic cusps. For simplicity, let us assume that $D C_{M}$ has exactly one cusp. The general case follows by performing the following geometric operations on each cusp individually.

Pick a compact exhaustion $K_{i}$ of $D C_{M}$ such that each boundary $\partial K_{i}$ is a smooth horospherical torus. By "opening up" the cusp of $D C_{M}$ one can construct a family of metric spaces $\left\{\left(A, d_{\varepsilon}\right)\right\}_{\varepsilon \in(0,1]}$ such that: 
1. $A$ is homeomorphic to $K_{i}$.

2. $\left(A, d_{\varepsilon}\right)$ is an Alexandrov space with curvature bounded below by $-1-c_{\varepsilon}^{2}$, where $\lim _{\varepsilon \rightarrow 0} c_{\varepsilon}=0$.

3. For each $i$, there is an isometric embedding $K_{i} \rightarrow\left(A, d_{\varepsilon}\right)$ for all sufficiently small $\varepsilon$.

4. $\lim _{\varepsilon \rightarrow 0} \operatorname{Vol}\left(A, d_{\varepsilon}\right)=\operatorname{Vol}\left(D C_{M}\right)$.

5. Near the boundary of $A$ the metric $d_{\varepsilon}$ is a flat Riemannian metric with totally geodesic boundary $\partial A$.

6. For any $\varepsilon \leq 1,\left(\partial A, d_{\varepsilon}\right)=\left(\partial A, \varepsilon \cdot d_{1}\right)$.

(A careful and clear explanation of this procedure is in [Bes, Section 2.2]. See also [L].)

Let $W$ denote a solid torus. Using the fact that $W$ is a product of a disk and a circle, one can build a product Riemannian metric $g$ on $W$ such that:

1. $(W, g)$ has totally geodesic boundary isometric to an Euclidean torus.

2. $(W, g)$ has sectional curvature bounded below by zero.

A manifold homemorphic to $L$ is obtained from $A$ by appropriately gluing $\partial W$ to $\partial A$ (i.e. by an appropriate Dehn surgery). The goal is to perform this gluing geometrically. To do so we must scale appropriately and interpolate between the flat torus boundaries of $\left(A, d_{\varepsilon}\right)$ and $(W, g)$. Let $T^{2} \times[0,1]$ denote a trivial interval bundle over a torus. Pick diffeomorphisms

$$
\phi_{0}: T^{2} \times\{0\} \rightarrow \partial A \text { and } \phi_{1}: T^{2} \times\{1\} \rightarrow \partial W
$$

such that the glued up manifold

$$
A \bigcup_{\phi_{0}}\left(T^{2} \times[0,1]\right) \bigcup_{\phi_{1}} W
$$

is homeomorphic to $L$.

Consider the metrics $\phi_{0}^{*} d_{\varepsilon}$ and $\phi_{1}^{*} g$ on the boundary of $T^{2} \times[0,1]$. We now apply a lemma from [Bes].

Lemma 8.4 ([Bes, Appendix A.2]). For any $n>0$ there exist $\alpha_{n}, \varepsilon_{n}>0$ and a Riemannian metric $\sigma_{n}$ on $T^{2} \times[0,1]$ such that:

1. The curvature of $\sigma_{n}$ is bounded between $-1 / n$ and $1 / n$.

2. The volume of $\left(T^{2} \times[0,1], \sigma_{n}\right)$ is less than $1 / n$.

3. $\left(T^{2} \times[0,1], \sigma_{n}\right)$ has totally geodesic boundary given by $\left(T^{2} \times\{0\}, \phi_{0}^{*} d_{\varepsilon_{n}}\right)$ and $\left(T^{2} \times\{1\}, \alpha_{n} \cdot \phi_{1}^{*} g\right)$.

4. $\alpha_{n}$ and $\varepsilon_{n}$ go to zero as $n \rightarrow \infty$. 
We may now glue geometrically to form

such that:

$$
\left(Y, \Delta_{n}\right):=\left(A, d_{\varepsilon_{n}}\right) \bigcup_{\phi_{0}}\left(T^{2} \times[0,1], \sigma_{n}\right) \bigcup_{\phi_{1}}\left(W, \alpha_{n} \cdot g\right),
$$

1. $\operatorname{Vol}\left(Y, \Delta_{n}\right) \rightarrow \operatorname{Vol}\left(D C_{M}\right)$.

2. $\left(Y, \Delta_{n}\right)$ is an Alexandrov space with curvature bounded below by $-1-c_{\varepsilon_{n}}^{2}$.

3. $Y$ is homeomorphic to the closed hyperbolic manifold $L$.

There is a sequence $\eta_{n} \rightarrow 1$ such that the homothetically scaled spaces $\left(Y, \eta_{n} \cdot \Delta_{n}\right)$ are Alexandrov spaces with curvature bounded below by -1 . Theorem 4.1 applied to $\left(Y, \eta_{n} \cdot \Delta_{n}\right)$ and $L$ yields the inequality

$$
\operatorname{Vol}\left(Y, \eta_{n} \cdot \Delta_{n}\right) \geq \operatorname{Vol}(L)
$$

Taking $n \rightarrow \infty$ yields the desired inequality

$$
\operatorname{Vol}\left(D C_{M}\right) \geq \operatorname{Vol}(L)
$$

Using the above geometric surgery arguments, we now prove that the inequality of Theorem 8.1 holds also for pared acylindrical manifolds.

Corollary 8.5. Let $(N, P)$ be a compact pared acylindrical 3-manifold. Let $\left(M_{g}, m_{g}\right)$ be a hyperbolic 3-manifold such that $C_{M_{g}}$ is homeomorphic to $N \backslash P$ and the boundary of the convex core $\partial C_{M_{g}} \subset M_{g}$ is totally geodesic. For all $(M, m) \in \mathrm{H}(N, P)$,

$$
\operatorname{Vol}\left(C_{M}\right) \geq \operatorname{Vol}\left(C_{M_{g}}\right) .
$$

Proof. $D C_{M_{g}}$ is a finite volume hyperbolic manifold. By repeating the arguments from the beginning of Case 2 above, it follows that $D C_{M_{g}}$ is obtained topologically by performing a (possibly empty) set of Dehn surgeries on the manifold $D C_{M}$. (Note that since $D C_{M_{g}}$ is not compact, Dehn surgery is not performed on every end of $D C_{M}$. The ends corresponding to the cusps of $D C_{M_{g}}$ are not changed.)

By performing an infinite sequence of hyperbolic Dehn surgeries on $D C_{M_{g}}$ we obtain a sequence of closed hyperbolic manifolds $L_{k}$ such that $\operatorname{Vol}\left(L_{k}\right) \nearrow \operatorname{Vol}\left(D C_{M_{g}}\right)$ [NZ]. For each $k$, a manifold homeomorphic to $L_{k}$ can be obtained from $D C_{M}$ by performing an appropriate topological Dehn surgery on each end of $D C_{M}$. By repeating the geometric surgery arguments of Case 2 above, the closed manifold $Y_{k}$ obtained by these Dehn surgeries on $D C_{M}$ can be given a sequence of metrics $\delta_{k}^{n}$ such that:

1. $\lim _{n \rightarrow \infty} \operatorname{Vol}\left(Y_{k}, \delta_{k}^{n}\right) \rightarrow \operatorname{Vol}\left(D C_{M}\right)$.

2. $\left(Y_{k}, \delta_{k}^{n}\right)$ is an Alexandrov space with curvature bounded below by -1 . 
3. $Y_{k}$ is homeomorphic to the closed hyperbolic manifold $L_{k}$.

Applying Theorem 4.1 to the sequence $\left\{\left(Y_{k}, \delta_{k}^{n}\right)\right\}_{n}$ and $L_{k}$ yields

$$
\operatorname{Vol}\left(D C_{M}\right) \geq \operatorname{Vol}\left(L_{k}\right) \text {. }
$$

Taking $k \rightarrow \infty$ yields

$$
\operatorname{Vol}\left(D C_{M}\right) \geq \operatorname{Vol}\left(D C_{M_{g}}\right)
$$

\subsection{Cone-manifolds}

Theorem 8.6. Let $Z$ be compact $n$-dimensional $(n \geq 3)$ cone-manifold built with simplices of constant curvature $K \geq-1$. Assume all its cone angles are $\leq 2 \pi$. Let $M_{\mathrm{hyp}}$ be a closed hyperbolic n-manifold. If $f: Z \rightarrow M_{\mathrm{hyp}}$ is a homotopy equivalence then

$$
\operatorname{Vol}(Z) \geq \operatorname{Vol}\left(M_{\text {hyp }}\right)
$$

with equality if and only if $f$ is homotopic to an isometry.

Proof. Since $K \geq-1$ and all the cone angles of $Z$ are $\leq 2 \pi$, this implies $Z$ is an Alexandrov space with curvature bounded below by -1 [BGP, p. 7]. Therefore, by Theorem 2.7, the volume growth entropy of $\widetilde{Z}$ is less than or equal to $(n-1)$. Applying Theorems 4.1 and 7.1 proves the theorem.

\subsection{Alexandrov spaces}

Theorem 8.7. Let $Z$ be a compact $n$-dimensional $(n \geq 3)$ Alexandrov space with curvature bounded below by -1 . Let $M_{\mathrm{hyp}}$ be a closed hyperbolic $n$-manifold. If $f: Z \rightarrow M_{\text {hyp }}$ is a homotopy equivalence then

$$
\operatorname{Vol}(Z) \geq \operatorname{Vol}\left(M_{\mathrm{hyp}}\right) .
$$

Proof. Otsu and Shioya proved that a finite dimensional Alexandrov space with curvature bounded below by -1 is almost everywhere Riemannian [OS]. By Theorem 2.7, the volume growth entropy of $\widetilde{Z}$ is $\leq(n-1)$. The theorem now follows from Theorem 4.1.

8.4. $n$-manifolds with boundary. The argument used in Section 8.1 generalizes immediately to prove a version of the Besson-Courtois-Gallot theorem for convex Riemannian $n$-manifolds with boundary. (For the definition of convex Riemannian manifolds with boundary, see Section 2.7.) A good example of a convex Riemannian manifold with boundary is a convex core with positive volume.

Perelman's Doubling theorem [P, Theorem 5.2] is used in the proof of Theorem 8.9. Unfortunately, the well known preprint $[\mathrm{P}]$ remains unpublished. 
Theorem 8.8 ([P, Theorem 5.2]). Metrically doubling any Alexandrov space with curvature bounded below by $k$ across its boundary produces an Alexandrov space with curvature bounded below by $k$.

Theorem 8.9. Let $Z$ be a compact convex Riemannian n-manifold with boundary $(n \geq 3)$. Assume the sectional curvature of $\operatorname{int}(Z)$ is bounded below by -1 . Let $Y_{\text {geod }}$ be a compact convex hyperbolic n-manifold with totally geodesic boundary. Let $f:(Z, \partial Z) \rightarrow\left(Y_{\text {geod }}, \partial Y_{\text {geod }}\right)$ be a homotopy equivalence of pairs. Then

$$
\operatorname{Vol}(Z) \geq \operatorname{Vol}\left(Y_{\text {geod }}\right),
$$

with equality if and only if $f$ is homotopic to an isometry.

Proof. The homotopy equivalence of a pairs $f:(Z, \partial Z) \rightarrow\left(Y_{\text {geod }}, \partial Y_{\text {geod }}\right)$ can be extended to a homotopy equivalence between the doubled manifolds $f: D Z \rightarrow$ $D Y_{\text {geod }}$. We know the sectional curvature of $\operatorname{int}(Z)$ is bounded below by -1 . So $Z$ is an Alexandrov space with curvature bounded below by -1 . By Perelman's Theorem $8.8, D Z$ is also an Alexandrov space with curvature bounded below by -1 . Theorem 2.7 then implies the volume growth entropy of $\widetilde{D Z}$ is not greater than $h\left(\mathbb{H}^{n}\right)=(n-1)$. Applying Theorem 4.1 yields the desired inequality.

Assume the inequality is an equality. Theorem 7.1 then implies the natural map $F: D Z \rightarrow D Y_{\text {geod }}$ is an isometry. As before, let $\sigma$ be the boundary preserving isometric involution of $D Z$ and $D Y_{\text {geod }}$. Since $f$ is $\sigma$-equivariant, $F$ is $\sigma$-equivariant (see Remark 4.2). Therefore, by Theorem 7.1, F:Z $\rightarrow Y_{\text {geod }}$ is an isometry.

\section{References}

[AMR] I. Aitchison, S. Matsumotoi, J. H. Rubinstein, Immersed surfaces in cubed manifolds. Asian J. Math. 1 (1) (1997), 85-95. Zbl 0935.57033 MR 1480991

[Bes] L. Bessières, Sur le volume minimal des variétés ouvertes. Ann. Inst. Fourier 50 (3) (2000), 965-980. Zbl 0971.53027 MR 1779901

[BBI] D. Burago, Y. Burago, and S. Ivanov, A Course in Metric Geometry. Grad. Stud. Math. 33, Amer. Math. Soc., Providence, R.I., 2001. Zbl 0981.51016 MR 1835418

[BCG1] G. Besson, G. Courtois, and S. Gallot, Entropies et rigidités des espaces localement symétriques de courbure strictement négative. Geom. Funct. Anal. 5 (5) (1995), 731-799. Zbl 0851.53032 MR 1354289

[BCG2] G. Besson, G. Courtois, and S. Gallot, Minimal entropy and Mostow's rigidity theorems. Ergodic Theory Dynam. Systems 16 (1996), 623-649. Zbl 0887.58030 MR 1406425

[BCS] J. Boland, C. Connell, and J. Souto, Minimal volume and minimal entropy. In J. Souto Clement Geometric structures on 3-manifolds and their deformations, Bonner Math. Schriften 342. Bonn 2001. Zbl 0998.57040 MR 1934287 
[BGP] Y. Burago, M. Gromov, and G. Perel'man, A. D. Aleksandrov spaces with curvatures bounded below. Uspekhi Mat. Nauk 47 (2) (1992), 3-51; English transl. Russian Math. Surveys 47 (2) (1992), 1-58. Zbl 0802.53018 MR 1185284

[BGS] W. Ballmann, M. Gromov, and V. Schroeder, Manifolds of nonpositive curvature. Progr. Math. 61, Birkhäuser, Boston, MA, 1985. Zbl 0591.53001 MR 0823981

[BM] M. Burger and S. Mozes, CAT(-1)-spaces, divergence groups and their commensurators. J. Amer. Math. Soc. 9 (1) (1996), 57-93. Zbl 0847.22004 MR 1325797

[Bon] F. Bonahon, A Schläfli-type formula for convex cores of hyperbolic 3-manifolds. $J$. Differential Geom. 50 (1998), 25-58. Zbl 0944.53021 MR 1678473

[BP] R. Benedetti and C. Petronio, Lectures on Hyperbolic Geometry. Universitext, Springer-Verlag, Berlin 1992. Zbl 0768.51018 MR 1219310

[CHK] D. Cooper, C. Hodgson, and S. Kerckhoff, Three-dimensional Orbifolds and ConeManifolds. MSJ Memoirs 5, Mathematical Society of Japan, Tokyo 2000. Zbl 0955.57014 MR 1778789

[EM] D. Epstein and A. Marden, Convex hulls in hyperbolic space, a theorem of Sullivan, and measured pleated surfaces. In Analytical and Geometric Aspects of Hyperbolic Spaces, London Math. Soc. Lecture Note Ser. 111, Cambridge University Press, Cambridge 1987, 3-92. Zbl 0612.57010 MR 0903852

[EG] L. Evans and R. Gariepy, Measure Theory and Fine Properties of Functions. Stud. Adv. Math., CRC Press, Boca Raton 1992. Zbl 0804.28001 MR 1158660

[F] R. Feres, The minimal entropy theorem and Mostow rigidity, after G. Besson, G. Courtois, and S. Gallot. Unpublished.

[GH] E. Ghys and P. de la Harpe (eds.), Sur les Groupes Hyperboliques d'après Mikhael Gromov. Progr. Math. 83, Birkhäuser, Boston, MA, 1990. Zbl 0731.20025 MR 1086648

[Gr] M. Gromov, Metric Structures for Riemannian and Non-Riemannian Spaces. Progr. Math. 152, Birkhäuser, Boston, MA, 1999. Zbl 0953.53002 MR 0682063

[J] W. Jaco, Lectures on Three-Manifold Topology. CBMS Reg. Conf. Ser. Math. 43, Amer. Math. Soc., Providence, R.I., 1980. Zbl 0433.57001 MR 0565450

[L] B. Leeb, 3-manifolds with(out) metrics of nonpositive curvature. Invent. Math. 122 (2) (1995), 277-289. Zbl 0840.53031 MR 1358977

[M] J. Morgan, On Thurston's uniformization theorem for three-dimensional manifolds. In The Smith Conjecture, ed. by J. Morgan and H. Bass, Pure Appl. Math. 112, Academic Press, Orlando, FL, 1984, 37-125. Zbl 0599.57002 MR 0758464

[NZ] W. Neumann and D. Zagier, Volumes of hyperbolic 3-manifolds. Topology 24 (3) (1985), 307-332. Zbl 0815482 MR 0589.57015

[OS] Y. Otsu and T. Shioya, The Riemannian structure of Alexandrov spaces. J. Differential Geom. 39 (1994), 629-658. Zbl 0808.53061 MR 1274133

[P] G. Perel'man, Alexandrov spaces with curvatures bounded from below II. Preprint, 1991.

[RS] M. Reed and B. Simon, Methods of modern mathematical physics. I: Functional analysis. Academic Press, New York 1980. Zbl 0459.46001 MR 0493419 
[S] P. Storm, Minimal volume Alexandrov spaces. J. Differential Geom. 61 (2002), 195-225. Zbl 1070.53023 MR 1972145

[Th1] W. Thurston, The topology and geometry of 3-manifolds. Princeton University, Lecture Notes, 1976-79.

[Th2] W. Thurston, Hyperbolic geometry and 3-manifolds. In Low-dimensional topology (Bangor, 1979), London Math. Soc. Lecture Note Ser. 48, Cambridge University Press, Cambridge, New York 1982, 9-25. Zbl 0483.57007 MR 0662424

[Th3] W. Thurston, Hyperbolic structures on 3-manifolds. I. Deformation of acylindrical manifolds. Ann. of Math. (2) 124 (2) (1986), 203-246. Zbl 0668.57015 MR 0855294

Received December 2, 2002; revised May 31, 2005

Peter A. Storm, Stanford University, Department of Mathematics, Bldg. 380, 450 Serra Mall, Stanford, CA 94305-2125, U.S.A.

E-mail: pastorm@stanford.edu 